\title{
Greening sample preparation: new solvents, new sorbents
}

\section{Ramos}

Department of Instrumental Analysis and Environmental Chemistry, Institute of Organic Chemistry, CSIC. Juan de la Cierva 3. 28006 Madrid.

* Corresponding author: L. Ramos

Phone: +34 915622900

Fax: +34915644853

Email: I.ramos@iqog.csic.es 


\begin{abstract}
Sample preparation is still identified as the bottle-neck of many modern analytical procedures due to the time-demanding nature of many of the treatment protocols in use. Sample treatment is also considered responsible for a large part of the analytical inaccuracy of the analytical methodologies because of the highly manipulative nature of most of these treatments. It is also one of the main limitations when trying to develop Green Analytical procedures due to the large amounts of reagents and energy consumption typically associated to most of the conventional sample preparation procedures. However, the efforts carried out during the last decades in this active research field are starting to revert the situation. Today, a plethora of miniaturized techniques are commercialized for the treatments of liquid (or dissolved) samples. When combined with an appropriated state-of-the-art separation-plus-detection technique, accurate analyte determination is possible even if only a very small amount of sample (i.e., few $\mathrm{mg}$ or $\mathrm{mL}$ ) is used for the analysis. More importantly, many of the these techniques allow sample preparation to be completed in a short time with minimum reagents and energy consumption, and with a significant reduction of the wastes generated. In recent years, initial limitations detected in some of these miniaturized solvent-based techniques are starting to be circumvented by the incorporation of new non-toxic extraction media as extractants. Similarly, sorbent-based techniques have benefited from advances in the field of engineered materials and nanotechnology by incorporation of novel sorbents with tuned physic-chemical properties for enhanced extraction efficiency and selectivity. Using the analysis of trace organic components in food and environmental matrices as case study, this book chapter reviews current state-of-the-art in the field of sample preparation, highlighting recent advances approaching the principles of Green Analytical Chemistry.
\end{abstract}

Keywords: Green Analytical Chemistry; Sample preparation; New sorbents; New solvents; Miniaturization 


\section{Introduction}

The term sample preparation refers to any type of treatment carried out to the representative subsample selected for analysis before final instrumental determination of the target compounds. The goal of the sample preparation step is consequently to render the investigated analyte to the measurement instrument in a form and concentration that allow its unambiguous identification and the accurate determination of its concentration in the original matrix. For obvious reasons, the probability to perform such a type of determination without any previous sample treatment decreases as the sample complexity increases and as the analyte concentration decreases, ${ }^{1}$ and becomes virtually impossible when the aim is the determination of trace organic compounds in highly complex matrices, such as most of the environmental and food samples. For such determinations, highly manipulative and time-consuming multistep procedures are still used for sample preparation, in particular when involving semi-solid and solid samples. ${ }^{2}$ In most cases, these conventional (i.e., large-scale) analytical procedures involve relatively large amounts of reagents and solvents, that should be subsequently evaporated and/or treated as toxic wastes. Efforts carried out in this field during the last decades to approach the principles of Green Chemistry have resulted in a plethora of novel (and frequently miniaturized) techniques and analytical methodologies, some of which were discussed in the original version of this book chapter ${ }^{3}$ and in a number of revision papers published since then. ${ }^{1,2,4}$ Despite progresses, significant differences remain in this field depending on the nature of the investigated sample. Thereby, while impressive advances have been achieved for the treatment of gaseous, liquid and viscous matrices, developments regarding (semi-)solid samples have been much more limited, most probably due to the lack of appropriate commercial instruments. ${ }^{2,4}$ In any case, and despite the many advances, the nature of the solvent used as extractant remains as a key aspect limiting the greenness of many of these methodologies.

This book chapter reviews developments and innovations introduced in the area of sample preparation during the last decade in line with previous considerations. To avoid as much as possible overlapping with the former version of this book ${ }^{3}$ and with other revision papers $\left(^{2,5-7}\right.$, among others) recently published, rather than presenting a comprehensive revision of all additions to the field, this chapter aims to present main analytical preparation techniques in use and their evolution during the last years. Special attention will be paid to current trends and possible lines of evolution in line with the principles of Green Analytical Sample Preparation ${ }^{1}$. In 
particular, the penetration of novel solvents and sorbents in the field of sample preparation and their impact and influence in greening of the treatment step will be discussed. Attention will focus on applications dealing with the analysis of minor (i.e., trace) organic components in complex matrices (e.g., food and environmental samples) due to the higher difficulty typically associated to this type of determination. Nonetheless, if relevant, application examples from close related areas will also be discussed as far as they involved chromatographic (or related separation) techniques for final instrumental determination. All chapter sections will start with a short description of the basis of the specific technique revised. Then, main developments and evolution trends observed in recent years will be identified and discussed through representative application studies. Examples dealing with the analysis of real samples will always be preferred.

\section{Solvent-based extraction techniques}

Despite its recognized shortcomings, viz. formation of emulsions, consumption of large volumes of organic (and frequently toxic) solvent(s) and dilution of the target compounds, ${ }^{8}$ liquid-liquid extraction (LLE) remains as the reference method of many well-established and official protocols. As for other sample preparation techniques, some of the practical limitations of LLE can be circumvented by simple scaling-down of the process. The miniaturization of the LLE process results, on one hand, in a significant reduction of solvent(s) and reagent(s) consumption, so reducing waste generation; but also on a faster phase separation and a more favorable phase ratio. All together yield more efficient, green and rapid analytical processes with improved analyte recoveries. ${ }^{2}$ When miniaturized LLE is, in addition, performed with a selective or green solvent alternative to most frequently use volatile organic solvents (VOSs), the technique results in a really advantageous, simple and cheap approach with many positive features. These considerations explain the increasing use of novel and tailored solvents, in particular ionic liquids (ILs), deep eutectic solvents (DESs) and natural deep eutectic solvents (NADESs), in combination with LLEbased techniques. ${ }^{9-11}$ However, it should be mentioned that, when these new solvents are used in an LLE format, their high viscosity made the stirring, heating ${ }^{12}$ or shaking (in many instances by the application of ultrasounds ${ }^{13}$ or bubbling) of the LLE mixtures frequently mandatory to speed the analyte partition process. Interestingly, the latter approach was used by Shishov et al. ${ }^{13}$ in a recent study reporting on the feasibility of performing this type of analyses in an automated and miniaturized manner. 
Miniaturized liquid-liquid partition is also the base of a plethora of solvent-microextraction (SME) techniques introduced essentially during the last two-three decades and whose principles and main application fields have been discussed in a number of recent reviews ${ }^{14-16}$ and one book. ${ }^{17}$ Current level of acceptation of SME-based techniques and formats for general use is rather variable. Single-drop microextraction (SDME), ${ }^{18}$ hollow fiber-protected solvent microextraction, both in its two/three-phase formats, $\mathrm{HF}(2 / 3) \mathrm{ME},{ }^{14}$ and dispersive liquid-liquid micro-extraction (DLLME) ${ }^{15,19}$ are among the most successful and widely accepted ones, probably due to the simplicity of their principles and instrumentation, the flexibility in their operational conditions and the possibility of obtaining ready-for-analysis extracts.

\subsection{Single-drop microextraction}

In SDME, a micro-drop of a water insoluble solvent (1-8 $\mu \mathrm{L}$ for VOSs and slightly larger for more viscous solvents) suspended at the tip of a gas chromatography (GC) syringe is either immersed in the investigated aqueous sample (typically, 1-10 mL) or exposed to the head-space (HS) of the vial that contains it until equilibrium is reached. The later approach, HS-SDME, is only feasible for the analysis of volatile non-polar analytes (or volatile non-polar analyte derivatives, which can be formed in-situ), but can be applied to gaseous, liquid or solid samples. The former approach, direct-immersion SDME (DI-SDME), has been demonstrated to be useful for the extraction of relatively non-polar and semivolatile analytes from clean aqueous samples. Meanwhile, its application to relatively complex samples is usually feasible only after sample filtration. In both cases, enrichment factors as large as 300 can be obtained. Once the extraction is completed, the micro-drop is withdrawn into the syringe and the concentrated extract directly subjected to

instrumental analysis, typically by GC. ${ }^{14,18}$ In SDME, diffusion governs the transport of the analyte from the drop surface to its inner part. Therefore, as far as the micro-drop was not dislodged or dissolved, the extraction time and efficiency can be favored by stirring, salting out or heating (including nebulization) $^{20}$ of the sample or, alternatively, by bubbling of a certain volume of air into the droplet. ${ }^{21}$

SDME of polar compounds is also possible but requires a modification that results in a three-phase system involving a liquid organic intermediate phase in which the neutralized polar analytes are extracted from the aqueous sample. The neutral analytes preconcentrated in this liquid membrane are then back-extracted into an aqueous micro-drop as polar compounds. This microdrop is finally withdrawn into a syringe for direct either liquid chromatography (LC) or capillary 
electrophoresis (CE) analysis. This three-phase configuration is usually named as liquid-liquidliquid microextraction (LLME). The higher stability provided to the extraction system by the intermediate liquid membrane allows the use of faster stirring rates: up to $1000 \mathrm{rpm}$ vs the 300 rpm typically used in the two-phase format at less a different type of set-up was used. ${ }^{22}$ In its turn, faster stirring results in shorter preconcentration times (typically, $15 \mathrm{~min}$ ), and improved enrichment factors (up to 500) compared to the two-phase SDME systems. In practice, the intermediate organic phase also acts as an organic liquid membrane allowing the simultaneous enrichment and clean-up of the analytes. Consequently, LLLME would be more appropriate for the analysis of relatively complex samples, such as human biological fluids, than the two-phase SDME format. ${ }^{14}$

Although automation continues being mentioned as one of the main limitations of SDME-based techniques, complete unattended sample preparation should be possible by using any of the modern multipurpose autosamplers nowadays commercially available. ${ }^{23,} 24$ In recent years, research in the field of SDME has been mainly orientated to explore the possibilities of using extractants alternative to the conventional VOSs used at early stages of the technique. ${ }^{2}$ Among them, ILs, ${ }^{9}$ supramolecular solvents ${ }^{25}$ and, more recently, DESs ${ }^{11}$ are the most representative examples. Selected application studies involving these and other alternative extractant systems for SDME are presented in Table 1.ILs are non-molecular solvents that remain as liquids at or near to room temperature (in general, defined as less than $150^{\circ} \mathrm{C}$ ) due to poor coordination of the ions..$^{\text {, }}$ ${ }^{45}$ ILs are characterized by their negligible volatility, high thermal stability and low flammability over a wide range of temperatures. These characteristics, together with their high viscosity, the possibility of tuning their physic-chemical characteristics by controlling the length and branching of the alkyl groups and/or the nature of the cation to which they are incorporated, and their capability to dissolve simultaneously compounds of very different nature, have contributed to expand their use as solvents in SDME in recent years. However, somehow surprisingly due to the constantly increasing number of ILs commercialized and synthesized, but in agreement to that observed for other extraction techniques, imidazolium-based ILs are clearly predominant in the application studies reported so far, even although these salts are known to absorb over the entire UV region. Also fluoride-containing anions are widely used (in particular, $\mathrm{PF}_{6}{ }^{-}$and $\mathrm{BF}_{4}{ }^{-}$) although, in the presence of moisture, these anions can produce $\mathrm{HF}$ and cause glassware and steel corrosion. ${ }^{46}$ In practice, usual extractant volumes in IL-based SDME studies are in the 4-10 $\mu \mathrm{L}$ range. However, the high viscosity of these salts (typically 2-3-folds above those of conventional organic solvents) 
should allow to use relatively large drops (up to $20 \mu \mathrm{L})^{47}$ without risk of dislodgement even if fast stirring rates or dynamic approaches are used..$^{48}$ In addition, the low volatility of ILs prevents from solvent volatilization during extraction, making possible to use higher extraction temperatures. All together results in higher enrichment factors, better extraction efficiencies and improved detectability with shorter extraction times compared to conventional SDME procedures. On the other hand, the high viscosity of ILs makes their handle with micro syringes difficult, a problem that has been solved by attaching a wider tube of a relatively inner material to the needle. ${ }^{49}$

Coupling IL-based SDME techniques to LC do not require any special interface or modification of the system. The short retention time of ILS in reversed-phase LC (in general, near the dead volume) favors the chromatographic separation of the IL from the target analytes and can be considered an extra advantage of this coupling. In contrats, the quoted above low volatility of ILs would prevent direct injection into GC systems. However, this problem can be solved through installation of home-made removable GC interfaces ${ }^{47}$ or by adaptation of a commercial one. ${ }^{49}$ Coupling between IL-SDME and other separation techniques (e.g., CE) ${ }^{30}$ or detection techniques (e.g., ion-mobility spectrometry) ${ }^{50}$ are still relatively rare in the literature.

Recent trends in IL-based DI-SDME include the use of more hydrophobic and hydrolytically-stable salts than those based on the use of $\mathrm{PF}_{6}{ }^{-}$as anion to minimize drop dissolution. ${ }^{51}$ More complex extraction mixtures have also being used in both HS- and DI-SDME to improve the extraction efficiency and selectivity when dealing with the analysis of trace components in complex matrices. In a recent illustrative example of the analytical potential of this type of approach, the feasibility of [BMIM][PF6] mixed with oxidized-nanofibers of cellulose (o-NC) and carboxylated carbonanotubes (c-CNT) for the fast and selective extraction of 2-amino-3,8-dimethylimidazo[4,5-f]quinoxaline from fried sausage was evaluated and probed to be superior to those observed when each component of the mixture was separately applied. ${ }^{30}$ The method provided quantitative extraction of the target compound in 30 min with a satisfactory repeatability (3\%) and reproducibility (4\%), adequate limits of quantitation (LOQs, $0.96 \mathrm{mg} / \mathrm{L}$ ) and a satisfactory linear response in the investigated range of 0.1-10 $\mathrm{mg} \mathrm{L}^{-1}$. No matrix interference was reported and the maximum interference levels tolerated for closely related compounds, such as 2-amino-3,4-8trimethylimidazo[4,5-f] quinoxaline and 4,5-b]pyridine, were $60 \%$ and $80 \%$, respectively.

The DESs, also so-called deep eutectic ILs, low-melting mixtures, or low transition temperature mixtures, ${ }^{11}$ could be considered as second-generation ILs. The DESs are composed of two or three cheap and non-toxic components capable of self-association, mainly through hydrogen bonds. ${ }^{52}$ 
The resulting eutectic fluid exhibits a melting point much lower than each of its individual components and, in general, remains as a liquid at temperatures below $130{ }^{\circ} \mathrm{C}$. DESs share a number of physico-chemical properties with the previously described ILs (i.e., low vapour pressure and flammability, chemical and thermal stability, and high viscosity), which made them feasible for the same types of applications. However, DESs are safe, biodegradable, and cheap because they can easily be prepared from accessible bulk chemicals. These extra remarkable features made DESs to attract researchers attention since their first introduction by Abbot et al. in $2001,{ }^{53}$ and they could also explain the increasing use of these green solvents in many application areas, in particular in food and pharmaceutical areas. ${ }^{54}$

In a representative early study, Tang et al. ${ }^{27}$ reported on the feasibility of $\mathrm{ChCl}$ :ethylene glycol for the HS-SDME of volatile bioactive terpenoids (linalool, a-terpineol and terpinyl acetate) from a slurry of Chamaecyparis obtuse leaves. In that study, a 2- $\mu \mathrm{L}$ droplet of $\mathrm{ChCl}$ :ethylene glycol (1:4) was exposed to the head space of a sealed vial containing $0.30 \mathrm{~g}$ of the dried and powdered plant leaves dissolved in $3 \mathrm{~mL}$ of methanol. The slurry was heated at $100{ }^{\circ} \mathrm{C}$ and the extraction extended for 30 min before any loss of the target terpenoids was reported. Then, the DES droplet was withdrawn into the GC syringe and subjected to GC-FID analysis without any extra treatment. Despite the simplicity of the method proposed, its efficiency for the preconcentration of the three studied terpenoids was superior to that obtained by alternative LLE- and USE-based methods used as reference methodologies.

Coacervates based on supramolecular assemblies (e.g., surfactant micelles) have also been evaluated as extractants in SDME. Coacervates can be considered as multifunctional solvents able to extract a wide variety of analytes due to the different interactions they can bring simultaneously. As an example, the feasibility of the approach for effective SDME of chlorophenols from water samples of different complexity was illustrated by Lopez-Jimenez et al. ${ }^{31}$ These researchers used tetrabutyl ammonium hydroxide to induce the formation of vesicular coavervates containing equimolecular amounts of decanoic acid and decanoate, and demonstrated that the microextraction with coacervates fit to the thermodynamic and kinetics derived for conventional organic solvents. Consequently, the experimental parameters affecting the extraction process were essentially similar in both cases and both approaches shared main advantages and limitations. However, the possibility of direct immersion of the drop in the water sample, with the consequent simplification of the extraction procedure, and the larger number of interactions possible in the case of vesicular coacervates (Figure 1) resulting in an improved 
detectability, were identified as specific advantages associated to the use of these types of supramolecular extractants.

All previously describe SDME-based techniques are static in nature and, in consequence, the main factor controlling the duration and efficiency of the extraction process is the diffusion of the extracted analytes. Although the use of less viscous solvents and higher stirring rates and temperatures can partially contribute to speed up the diffusion of the target compounds from the drop surface to its inner part, constant renovation of the drop surface by using a dynamic approach is probably a more effective approach. Up to now, two types of dynamic SDME approaches have been described: in-syringe and in-needle SME. In the in-syringe approach, the aqueous sample or HS is repeatedly withdrawn and ejected into the syringe needle or lumen containing the receiving organic phase. ${ }^{55}$ On the contrary, in the in-needle approach, around $90 \%$ of the extraction drop is withdrawn into the syringe needle and then pushed out again repeatedly for sample exposure. ${ }^{56}$ The in-needle approach may be feasible for the treatment of samples containing relatively high amount of matrix components that could affect the subsequent instrumental analysis. Meanwhile, the in-syringe approach is somehow limited to the treatment of relatively pristine samples. Interestingly, a modification of this later approach has been used for the simultaneous in-syringe derivatization and preconcentration of aliphatic amines from alkalized aqueous samples. ${ }^{24}$ The method, involving an LC-FLD for analyte instrumental determination, provided limits of detection (LODs) below $19 \mathrm{ng} \mathrm{mL}^{-1}$ and a linear response in the evaluated range of $25-500 \mu \mathrm{g} \mathrm{L}^{-1}$.

\subsection{Hollow fiber-protected two/three-phase solvent microextraction}

In its simplest version, the hollow fiber-protected two-phase solvent microextraction, $\mathrm{HF}(2) \mathrm{ME}$, technique involves a small-diameter microporous polypropylene tube (the hollow fiber), usually sealed at one end, to contain the organic solvent used as extractant. The open end of the hollow fiber is attached to a syringe needle used to fill the fiber with $4-12 \mu \mathrm{L}$ of the extraction solvent (e.g., toluene, undecane, 1-octanol, or dihexyl ether). Then, the fiber is immersed in the investigated aqueous sample for a preselected time (ca. 20-60 min) to allow the (more or less) hydrophobic target analytes to migrate through the wall pores into the solvent. Once the extraction is completed, the enriched solvent is withdrawn with the syringe and directly subjected to instrumental analysis, in general, by GC. HF(2)ME can be considered a liquid-liquid membrane 
extraction and, consequently, it would be more adequate that its equivalent two-phase SDME technique for the treatment of "dirty" aqueous samples. Due to the stability of the system, fast stirring is possible, which in combination with the use of relatively large extractant volumes, results in higher enrichment factors compared to SDME. On the contrary, the longer extraction times required to complete the extraction process and the fact that only a fraction of the total enriched solvent is used for final determination are usually considered shortcomings of this technique compared to SDME. Although HF(2)ME can be adapted for use with an autosampler, ${ }^{56}$ but every fiber should be manually sized and prepared before use, which is probably the main practical limitation of the technique.

$\mathrm{HF}(3) \mathrm{ME}$ is operated in a similar way to $\mathrm{HF}(2) \mathrm{ME}$ but in this case the water-immiscible organic solvent fills the pores of the hollow fiber polymer and a third phase, an aqueous acceptor phase, is used to fill the fiber lumen. Transfer of the target analytes through the three phases involved in the extraction process is controlled through $\mathrm{pH}$ changes and, as the final acceptor phase is aqueous, LC or CE are usually preferred for final instrumental determination of the investigated compounds.

Up to now, dynamic versions of $\mathrm{HF}(2) \mathrm{ME}$ has received limited attention, ${ }^{57,58}$ probably due to the higher complexity of the approach. The same consideration applies for some alternative configurations for $\mathrm{HF}(2 / 3) \mathrm{ME}$, such as $\mathrm{SBME}^{59}$ in which the fiber is sealed at both ends, which allows its complete immersion into the stirred solution and results in an improved extraction efficiency. ${ }^{60}$ The larger sample volume required for SBME compared to $\mathrm{HF}(2 / 3) \mathrm{ME}$ could be a possible explanation for this observation.

$\mathrm{HF}(2 / 3) \mathrm{ME}$ is particularly suited for the treatment of size-limited aqueous samples and, at present, one of its more active application areas is the treatment of biological fluids. The polar nature of most of the analytes investigated in this research area made possible to favor the HF(3)ME process by the application of a potential difference between the sample and the aqueous acceptor phase. This technique is referred as electromembrane extraction (EME). EME is intended for charged molecules and involves electrokinetic migration of the analytes through a supported liquid membrane. The membrane prevents major matrix components to reach the acceptor phase and so EME would be more adequate for the treatment of complex biological and environmental samples than HF(3)ME. The application of an electrical potential increases significantly the mass transfer through the membrane and typically reduces the extraction time from ca. $45 \mathrm{~min}$ in $\mathrm{HF}(3) \mathrm{ME}$ to some $5 \mathrm{~min}$. EME basis, recent innovations, including on-chip EME ${ }^{61}$ and its 
hyphenation with UV and MS detectors, ${ }^{62,63}$ as well as the most relevant application areas of this technique have been discussed in several recent reviews to which the reader is addressed for further reading. ${ }^{14,24,64}$ Today, the commercialization of appropriate equipment (including portable instruments) and the still limited understanding of the different parameters affecting the analytes electromigration through the supported membrane ${ }^{48}$ remain as the main limitations of this anyway interesting, economic, selective, rapid and efficient novel technique.

In recent years, efforts have been made to improve the selectivity and efficiency of the $\mathrm{HF}(2 / 3) \mathrm{ME}$ process by modifying the nature of the membrane by using alternative extractants. This made increase the interest for ILs as solvents in $\mathrm{HF}(2 / 3) \mathrm{ME}$. The high affinity of polar analytes for ILs has been demonstrated to be an advantageous feature in $\mathrm{HF}(2) \mathrm{ME}$ yielding high enrichment factors and improved selectivity compare to conventional organic solvents. ${ }^{29}$ In HF(3)MS, ILs have typically been used as intermediate solvent owing their immiscibility in the aqueous sample and the organic acceptor phase. Under this configuration, ILs look to be particularly interesting in applications dealing with the simultaneous extraction of non-polar and polar analytes from relative complex aqueous matrices (Table 1 ). In this sense, Tao et al. ${ }^{33}$ illustrated the feasibility of $\mathrm{HF}(3) \mathrm{MS}$ using $\left[\mathrm{C}_{8} \mathrm{MIM}\right]\left[\mathrm{PF}_{6}\right]$ modified with $14 \%$ tri-n-octylphosphine oxide (TOPO) (w/v) as organic liquid membrane and alkaline water $(\mathrm{pH} \mathrm{13,} \mathrm{NaOH})$ as acceptor phase for quantitative extraction of sulfonamides from river and farm water at $\mathrm{pH}$ 4.5. The selectivity of the extraction process was demonstrated by successful analysis in presence of up to $25 \mathrm{mg} \mathrm{L}^{-1}$ of humic acid and up to $100 \mathrm{~g}$ $\mathrm{mL}^{-1}$ of bovine serum albumin. The low LODs (0.1-0.4 $\mathrm{g} \mathrm{L}^{-1}$ ) reported for test compounds (i.e., sulfadiazine, sulfamerazine, sulfamethazine, sulfadimethoxine and sulfamethoxazole) using LC-UV and the satisfactory repeatability (RSDs better than 7\%) of the procedure sharply contrasted with the $8 \mathrm{~h}$ required to compete the extraction process.

Carbon nanotubes (CNTs) have also been investigated as alternative extractant media with improved selectivity and efficiency. ${ }^{39,62,63}$ Multiwalled carbon nanotubes (MWCNTs) dispersed in 1-octanol were used to fill the wall pores of a polypropylene hollow fiber membrane used in a $\mathrm{HF}(3) \mathrm{ME}$ system employed for the extraction of caffeic acid from Echinacea purpurea herbal extracts. $^{62}$ The membrane remained stable in between the two aqueous phases, the sample and the acceptor buffer; and the analytes were simultaneous sorbed by the nanotubes and the dispersant solvent. Extraction was completed in $25 \mathrm{~min}$ and enrichment factors above 2000 were reported. In a subsequent closely-related study, the feasibility of this extractant mixture for $\mathrm{HF}(2) \mathrm{ME}$ of brilliant green from fish pond water was demonstrated. ${ }^{63}$ In this case, the extraction 
was performed in the format of SBME. Quantitative recovery (120\%), good repeatability (7\%) and an enrichment factor of 799 were reported after $30 \mathrm{~min}$ of extraction and using only $5 \mathrm{~mL}$ of sample and $3 \mu \mathrm{L}$ of acceptor phase. Examples on the analytical potential of SBME when using ILs ${ }^{34}$ and magnetized-ILs ${ }^{65}$ as extraction solvents have also been reported in the specialized literature.

\subsection{Dispersive liquid-liquid micro-extraction}

Since its introduction in $2006,{ }^{66}$ DLLME has experienced a rapid evolution and today it is considered a well-accepted technique, in particular for the treatment of liquid samples and extracts. ${ }^{15}$ DLLME was originally introduced as a modified miniaturized LLE in which a small volume of a water-immiscible solvent (typically 10-50 $\mu \mathrm{L}$ ) was dissolved in 0.5-2 $\mathrm{mL}$ of a water-miscible solvent being the mixture rapidly injected into the investigated aqueous sample (up to $10 \mathrm{~mL}$ ). The fast injection of this mixture of organic solvents into the sample made the water-immiscible solvent to be dispersed in the aqueous mass as small micro-drops. The promoted increase of the interfacial area between both phases makes analyte phase transition into the organic extractant to be fast and the equilibrium state to be quickly reached. The enriched organic phase is finally separated from the aqueous phase by either centrifugation or frozen (depending on its density) and, then, directly subjected to instrumental analysis (in general, by GC). As initially proposed, the technique was suited for the enrichment of non-polar analytes from pristine aqueous samples. Its application to the analysis of polar analytes required previous water $\mathrm{pH}$ adjustment and/or analyte derivatization, the latter being usually performed in-situ or, preferably, by dispersion of the derivatization agent together with the extractant. ${ }^{15,19,67}$ The potential of DLLME for the preconcentration and/or clean-up of analytes from diluted (in general, aqueous) extracts obtained from (semi-)solid matrices has also been demonstrated by a number studies. ${ }^{68-70}$

Whatever the goal of the analysis, DLLME can be considered a green, simple, fast and efficient extraction and preconcentration technique (enrichment factors in the 100-900 range). However, it is also a highly manipulative procedure for which even partial automation is difficult. In any case, since its introduction one decade ago, DLLME has experienced a fast evolution and different modifications to the conventional approach have been introduced to promote further simplification of the process and/or improved efficiency and selectively, as it will be illustrated in the next paragraphs.

The dispersive solvent can decrease the partition coefficient of analytes into the extraction solvent and also complicate subsequent phase separation. ${ }^{71}$ However, its use can be avoided by 
application of an appropriate emulsifier force to the extraction system, for instances, by vortex shaking or ultrasonic irradiation. ${ }^{72}$ Vortexing promotes a mild emulsification effect that has been proved to be sufficient, for example, for the quantitative extraction of chloropyrifos and five pyrethroids from snow water (recoveries, $72-102 \%$; RSD better than $11 \%){ }^{73}$ In this study, $30 \mu \mathrm{L}$ toluene were used as extraction solvent and added to $20 \mathrm{~mL}$ of sample. No salt was added and the mixture was vortexed for $1 \mathrm{~min}$. An $1-\mu \mathrm{L}$ of the floated solvent was used for subsequent GCelectron capture microdetector (GC- $\mu \mathrm{ECD}$ ) for analyte determination. LODs of 3-10 $\mathrm{ng} \mathrm{L}^{-1}$ and enrichment factors of 835-1115 were obtained. When ultrasounds are applied, emulsification is achieved through cavitation, which is a more energetic process that breaks down the dispersed extractant drops and generate smaller droplets immediately after disruption. ${ }^{74}$ The efficiency of this emulsification process and the risk of analyte degradation (especially when using ultrasonic probes) makes that short ultrasonication times (typically, a few min) were generally applied in this type of procedure.

The range of high-density conventional organic solvents used in DLLME is essentially limited to (not-really-environmentally-friendly) chlorinated solvents. Thereby, the interest for using alternative low-density solvents resulted in the development of new DLLME approaches and novel extraction vessels. ${ }^{75}$ In an interesting study involving a novel type of extraction vessel, the efficiency of high- and low-density organic solvents for DLLME of a broad range of pharmaceuticals using ultrasonic assisted emulsification were compared. ${ }^{71}$ Unfortunately, experimental results demonstrated that, under the experimental conditions proposed, high-density solvents exhibited better performance and reproducibility values than low-density solvents. The authors concluded that the viscosity and interfacial tension of the solvent have a profound effect on the method performance, and that its boiling point and solubility determined the volume of extractant collected after DLLME, a variable that affected the reproducibility of the procedure. On the other hand, despite the satisfactory results obtained for the analysis of aqueous sample, the method failed in its application to more complex matrices, such urine and plasma, due to matrix precipitation. None of the pretreatment procedures assayed to reduced matrix complexity before DLLME contributed to improve this negative finding, something that evidenced the limitations of this technique for direct analysis of relatively complex matrices. As previously indicated, in these cases, DLLME usually performs better as preconcentration and/or purification technique. 
During the last years, DLLME has been greatly benefited from the use of alternative solvents providing improved extraction efficiency and selectivity ${ }^{76}$. Some selected IL- and DES-based DLLME application studies have been summarized in Table 1.

Hydrophobic ILs, dispersed ${ }^{35-37}$ or not ${ }^{38,39}$ in a co-solvent, have been used for the fast, green, simple and miniaturized extraction of heterocyclic insecticides ${ }^{35}$ or aromatic amines ${ }^{38}$ from aqueous samples, sudan I-IV from diluted red wine and fruit juices, ${ }^{39}$ or PCBs and PBDEs from acidified and filtered water and urine samples. ${ }^{36}$ Strategies to promote IL emulsification when the disperser is not used include fast reinjection of the IL-water mixture into the investigated sample, ${ }^{38}$ heating of the mixture, ${ }^{36,37}$ and the application of an auxiliary energy ${ }^{37}$ in a manner similar to that previously described for conventional organic solvents. Despite the efficiency of these emulsification approaches, the application of the required additional energy for heating, cooling and/or shaking of the sample represents an extra treatment to be incorporated to the analytical protocol. Up to now, three different procedures have been described to avoid these time-consuming steps. The first one was in-situ solvent formation, which simultaneously avoided the use of a disperser and contributed to improve the efficiency of the extraction process due to the larger contact surface between the extraction solvent and the aqueous sample. ${ }^{77}$ The second one consisted on the addition of unmodified magnetic nanoparticles to the IL-aqueous sample to retrieve and separate the enriched IL from the mixture. ${ }^{78}$ Once the supernatant was removed, the IL was desorbed from the nanoparticles by washing with an appropriate solvent and directly subjected to instrumental analysis. The third approach is the so-called in-syringe IL-based DLLME and required a simple plastic syringe as extraction unit. In this case, the extractant was rapidly sprayed into the aqueous sample, which was contained in a $10 \mathrm{~mL}$ syringe unit, to promote fast emulsion and analyte extraction. Then, the plunger of the syringe was slowly moved to the initial point allowing the recovery of the IL from the wall and the lower part of the syringe while the analysed aqueous sample was ejected from the unit. Finally, the enriched IL phase was recovered from the syringe tip and subjected to instrumental analysis. The technique was fast, simple, avoided the time-consuming centrifugation step, and had potential for automation. Its main limitation was the difficulty of complete recovery of the IL (in the original application example, only $30-40 \%$ of the IL was recovered), ${ }^{44}$ which could negatively affect the reproducibility of the process. Up to now, this technique has been used, for example, for the extraction of non-steroidal anti-inflammatory drugs from acidified and filtrated urine, ${ }^{44}$ benzoylurea insecticides from water 
and tea samples, ${ }^{79}$ and sulfonamides from serum, ${ }^{80}$ this latter application requiring the application of ultrasounds for IL emulsification and subsequent cooling of the sample for IL recovery.

The feasibility of other solvents, such as DESs, ${ }^{40,41}$ magnetofluids, ${ }^{81}$ or supramolecular systems, ${ }^{42,}$ 43, 82 for DLLME has also been evaluated in a number of interesting application studies. In general, the approaches used in these investigations are similar to those previously described for DLLME with magnetic nanoparticles and/or ILs, and shared with them advantages and limitations. However, those involving DESs and supramolecular systems are usually characterized by short extraction times (viz. 1-2 $\mathrm{min}$ ), the possibility of extracting hydrophilic analytes over a wide range of polarities and avoid the use of toxic solvents. ${ }^{6,11}$ On the other hand, centrifugation of the mixture is frequently mandatory to promote phase separation (although the possibility of frozen of the mixture has also been assayed).$^{40}$ As a typical example of the enhanced selectivity and improved enrichment provided by DLLME with these green solvents, Figure 2 shows the LCDAD/ESI-MS chromatograms obtained for a lake water and a 1:1 (v/v) diluted apple juice before and after spiking at the $0.1 \mu \mathrm{g} \mathrm{L}^{-1}$ level with diethofencarb and pyrimethanil and UA-DLLME with $0.05 \mathrm{mg}$ of Teew 80 . In this case, $20 \mu \mathrm{L}$ of carbon tetrachloride were also added to the extraction solution, which was ultrasonicated for $3 \mathrm{~min}$ at $25^{\circ} \mathrm{C}$. Then, the emulsion was disrupted by 2 min centrifugation at $350 \mathrm{rpm}$. The organic phase was sedimented at the bottom of the conical centrifuge tube $(10 \pm 1 \mu \mathrm{L})$ and collected for instrumental analysis of the two investigated fungicides. The method provided recoveries in the $86-115 \%$ range, RSDs better than $8 \%$, linear response in the evaluated range of $0.05-2000 \mu \mathrm{g} \mathrm{L}^{-1}$, and low LODs $\left(0.01 \mu \mathrm{g} \mathrm{L}^{-1}\right)$. Its potential for the analysis of real matrices was illustrated by successful application to naturally contaminated waters, including tap, lake and waste water samples. ${ }^{42}$

It is expected that the future development of novel (preferably green) solvents and supramolecular systems with improved extraction capabilities will contribute to expand the application fields of DLLME in coming years.

\section{Sorbent-based extraction techniques}

Most of the main miniaturized sorbent-based extraction techniques in use in laboratories, namely miniaturized solid-phase extraction (SPE), solid-phase microextraction (SPME), and stir-barsorptive extraction (SBSE), were developed years ago and, at present, they can be considered well- 
establish and accepted procedures for the treatment of gaseous, liquid, viscous and solid samples and extracts. During the last years, conceptual additions to this field have been rather limited. However, research concerning these techniques remains active through the development of novel sorbent with improved features regarding selectivity, loading capacity or retention efficiency, with special focus on analytes that were only slightly retained in previously available materials. Thereby, sorbent-based extraction techniques have benefited from the advances achieved during the last decade in other research areas, including the development of fine-tune solvents, engineered materials and nanotechnology. In addition, some novel formats and configuration have been introduced. Efforts have focused on the setting-up of systems allowing either hyphenation between the extraction technique and the chromatographic instrument used for final analyte determination, or to increase sample throughput. The feasibility of some techniques for insitu and in-vivo sampling has also been evaluated.

This section reviews the most relevant advances and main achievements reported in the last years in the field of sorbent-based microextraction on the light of previous considerations.

\subsection{Miniaturized solid-phase extraction}

On-line SPE is a well-established technique that is routinely used in many laboratories for the preconcentration and clean-up of analytes of different nature from aqueous samples. ${ }^{83}$ On-line SPE is typically performed by inserting a short stainless steel pre-column $(10-20 \mathrm{~mm} \times 1-4.6 \mathrm{~mm}$ I.D.) or a miniaturized SPE cartridge ( $10 \mathrm{~mm} \times 1-2 \mathrm{~mm}$ I.D.) packed with the appropriated sorbent in a valves-system. After preconcentration and (when required) drying of the cartridge, analytes are eluted from the sorbent with a small amount of an appropriate solvent (ca. 50-100 $\mu \mathrm{L}$ ) and directly transferred to the instrument selected for separation-plus-detection of the target analytes. In most applications dealing with the analysis of organic compounds, $10 \mathrm{~mL}$ of the investigated aqueous sample suffice for the accurate and sensitive determination of the analytes at trace level even for complex matrices such as waste water. Nevertheless, depending on the sorbent and the selected detection system, volumes as small as $1 \mathrm{~mL}^{84}$ or as large as $100 \mathrm{~mL}$ of sewage water ( $500 \mathrm{~mL}$ for pure water) can be loaded without breakthrough problems. ${ }^{85}$ The entire process takes place in a closed system, which minimizes sample and solvent consumption, avoids sample manipulation and facilitates automated and unattended work. Specific details regarding the on-line coupling of SPE with different chromatographic techniques and detectors have been 
discussed in more specific reviews to which the interested reader is addressed $\left(^{5,86,87}\right.$ and references therein).

Many types of SPE packing materials are nowadays commercialised and many other are synthesized "in-house" to fulfil the demands of specific application studies. The choice of the SPE sorbent must consider the nature of both the target compounds and the investigated sample and, in the case of on-line SPE, its compatibility with the analytical column phase.

Silica and silica-bonded materials are still the most commonly used sorbent in SPE, including online systems. Depending on the nature of the bonded group, packing materials are classified as reversed-phase sorbents ( $\mathrm{C} 8$ and $\mathrm{C} 18$ ), normal-phase sorbents (with e.g., $\mathrm{NH}_{2}$ and $\mathrm{CN}$ ), or ionexchange sorbents (either cationic or anionic). Other relevant sorbents include carbon-based sorbents and high capacity porous polymers, primarily the macroporous poly(styrendivinylbenzene) and, in particular, its different modifications with enhanced capacity (hypercrosslinked sorbents), polarity (hydrophilic macroporous and hydrophilic hypercrosslinked sorbents), or both simultaneously (dual-phase or mixed-mode sorbents). The improved features of the later sorbents make them particularly suitable for the accurate isolation of polar and ionic species from highly complex matrices through proper $\mathrm{pH}$ control of the matrix and desorptionsolution(s). ${ }^{87,} 88$ The analysis of trace pharmaceuticals in wastewater, ${ }^{85}$ drugs and metabolites in plasma, ${ }^{89}$ or amyloid $\beta$-peptides (early markers of Alzheimer's disease) in cerebrospinal fluid ${ }^{90}$ would be representative application examples of this type of analytical approach. Alternatively, when dealing with the treatment of very complex matrices containing large amounts of interfering species, compound-specific and class-specific sorbents based on molecular recognition, e.g. immunosorbents, molecularly-imprinted materials (MIPs) and the more recently introduced aptamer-modified surfaces, should be preferred. ${ }^{91-93}$ Compared to MIPs and immunosorbents, the preparation of aptamer-modified sorbents is fast and relatively inexpensive, avoids the use of animals, and requires only minute amounts of the analyte used as template. Recognition can be equally specific and the approach can be applied to both large (e.g., proteins) ${ }^{94}$ and small (e.g., ochratoxin A) ${ }^{95}$ molecules. Despite their many positive features, the number of application studies involving the use of aptamers as recognition element is still relatively limited in the literature.

Immobilization of an IL onto a silica- or polymer-based support confers the sorbent multi-modal type interactions that have been exploited in a number of applications during the last years. ${ }^{9,45,96}$ These materials showed a satisfactory loading capacity (up to $1 \mathrm{~L}$ ) and a selectivity similar to or better than that provided by conventional sorbents. In addition, polymer-based IL-SPE are stable 
through the entire $\mathrm{pH}$ range (unlike silica-based IL-SPE sorbents, which are typically limited to the 2-8 range). Detailed description on the different synthetic procedures used for their preparation, in-deep discussion of the possible mechanisms governing IL retention on the sorbent support and subsequent analyte retention in IL-based materials, as well as the most appropriate activation protocols can be found in texts of a more specific nature. ${ }^{45,96}$

Most of the IL-SPE sorbents synthesized up to now are based on imidazolium-based salts. When applied to the analysis of ionizable and, in particular, anionic compounds, these functional groups promote $\pi-\pi$ and anion-exchange interaction, the intensity of the later depending on the nature of the substitution and the length of the alkyl chain. ${ }^{97}$ Nevertheless, the nature of the anion has been found to play also a relevant role in the fine-tuning of the retention mechanism. For example, different extraction efficiencies were observed during preconcentration of acidic pharmaceuticals from environmental waters in a series of IL-SPE based, i.e. VDC-DVB[MIM][BF 4 , VDC$\mathrm{DVB}[\mathrm{MIM}]\left[\mathrm{CF}_{3} \mathrm{COO}\right]$ and $\mathrm{VDC}-\mathrm{DVB}[\mathrm{MIM}]\left[\mathrm{CF}_{3} \mathrm{SO}_{3}\right]{ }^{98}$ While similar satisfactory results were obtained with the two latter sorbents, the one containing $\left[\mathrm{BF}_{4}\right]$ showed poorer retention capability. The higher charge density in this ion compared to the other two was suggested to promote a stronger interaction with the imidazolium groups, which resulted in a reduction of the interactions with the test analytes.

Although anionic exchange in combination with reverse-phase interaction looks to be the most rational mechanism for IL-based $\mathrm{SPE}{ }^{96}$ these materials has also been applied to the preconcentration of analytes that did not contain any ionizable functional group. In this case, hydrophobic interactions looked to become dominant, as illustrated for the SPE of 12 sulfonylureas herbicides from water and acidified soil extracts using a novel material obtained by chemical immobilization of SilpR[MIM][PF6] on silica gel as sorbent. ${ }^{99}$ Although essentially similar, and relatively large, recovery ranges were reported for both matrices $(54-118 \%$ and $61-121 \%$ for water and soil extracts, respectively), the IL-based SPE procedure showed a satisfactory repeatability (RSD better than $11 \%$ in all cases), and an improved selectivity compared to the commercial C18 SPE cartridges.

Equivalent studies reporting of the use of DESs and NADESs grafted on the surface of specific sorbents to yield new materials with improved properties are still limited in the literature. In an early exploratory study, Gan et al. ${ }^{100}$ reported on the satisfactory performance of an anion exchange resin grafted with $\mathrm{ChCl}$ :glycerol (1:2) for the selective trapping of a neutral aromatic diterpene, cleistanthol, from Phyllanthus flexuosus root extracts (recovery, 82 \%) compared with 
those observed for the raw sorbent (68\%) and conventional C18 (72\%). Apart from this enhanced retention capability, the novel sorbent showed a improved selectivity for the target compounds compared to the other two evaluated materials. Similarly, the modification of the graphene surface with $\mathrm{ChCl}$ :ethylene glycol (1:1) showed a superior selectivity and a wrinkled structure that resulted in enhanced sorbent capabilities during the determination of sulfamerazine from river water than observed for the raw graphene. The high loading capacity of this novel DES-modified sorbent (18.62 $\mathrm{mg} \mathrm{g}^{-1}$ ) made that $2 \mathrm{mg}$ of the material sufficed for the intended SPE determination, which was performed in a pipette tip-format. ${ }^{101}$

Interestingly, in a recent study dealing with the SPE of bioactive compounds from extracts obtained from Artemisa Scopariae, the material resulting from the modification of the surface of a hybrid molecular imprinted polymer (MIP) with a ChCl:glycerol (1:3), provided better recoveries of the target compounds (i.e., rutin and quercetin) than when the MIP surface was modified with ILs based on 1-methylimidazole. ${ }^{102}$ In a follow up study, the same authors observed that the use of a ternary DES mixture (methyltriphenylphosphonium bromide:chalcone:formic acid, 1:0.1:2) for the DES-MISPE process ${ }^{103}$ contributed to reduce the viscosity and melting point of the original binary DES, while providing similar (for rutin, $92 \%$ ) or better (for quercetin, $94 \%$ vs $80 \%$ ) recoveries of the investigated analytes. These results illustrated the potential of some these novel DES-modified sorbents for selective preconcentration of minor compounds from complex extracts and open new perspectives regarding the development of innovative SPE materials with improved features.

Ionic surfactants can also be sorbed on the surface of active solids such as alumina, silica, titania, iron oxides and nanoparticles, as monolayers or bilayers. ${ }^{104}$ In the former structure, the hydrophobic tail of the surfactant is exposed to the sample solution. Thereby, the obtained sorbent, called hemimicelle, would exhibit affinity towards non-polar analytes. On the contrary, in the bilayer structure, called admicelle, the ionic tails of the surfactant are exposed to the sample, making the sorbent more suitable for the preconcentration (i.e., adsolubilization) of polar species. In any case, one of the most interesting features of these types of sorbents is their capability to simultaneously extract analytes of divergent polarities and natures due to their amphoteric nature. Alumina modified with admicelles of sodium dodecyl sulphate (SDS) and tetrabutylammonium (TBA) was used as a mixed-mode sorbent to extract and preserve pesticides with different functionalities from river and underground water. Triazines, carbamates, phenylureas, anilides, chloroacetanilides, organophosphorus and phenoxyacids were considered in 
the study. Despite the well-known instability of most of these analytes, no degradation was reported for a larger majority of the investigated pesticides after three months of storage of the cartridges at $-20^{\circ} \mathrm{C}$ in the darkness (recoveries in the $70-100 \%$ and RSDs better than $8 \%$, except for atrazine and simazine). Interestingly, up to $250 \mathrm{~mL}$ of sample were preconcentrated on the cartridges, while analyte elution was performed with only $1 \mathrm{~mL}$ of THF for basic and neutral pesticides, followed by $2 \mathrm{~mL}$ of $0.3 \mathrm{M} \mathrm{NaOH}$ :methanol $(90: 10, \mathrm{v} / \mathrm{v})$ for the subsequent separate elution of acidic pesticides.

Electrospun polymer nanofibers (NFs) and CNTs have also been evaluated as SPE sorbents. ${ }^{83,} 105$ The large surface areas of these nanomaterials resulted in a very large surface-to-volume ratio, which turned in improved retention capacities even if only a few mg of sorbent were used for SPE. The possibility of improving the selectivity of these nanomaterials by modifying their surface is another interesting feature that has been exploited in several recent application studies. For NFs, typical representative examples include the use of polyamide NFs for the on-line SPE of clodinafop propargyl from water, soil and wheat samples, ${ }^{106}$ and the development of hybrid composites by immobilizing aptamers specific for the protein thrombin on a polymeric polystyrene-poly(styreneco-maleic anhydride) nanofiber. ${ }^{107}$ CNTs have been used for the analysis of non-polar analytes, for which this sorbent shows a selectivity similar to that observed for other carbon-based materials, but with improved concentration capacity. (As an indication, it can be mentioned that MWCNPS are able to adsorb up to $10^{34}$ more 2,3,7,8-tetrachorodibenzo-p-dioxin than conventional carbon.) ${ }^{108}$ CNTs can also retain highly polar and ionic compounds and its surface can be easily modified by chemical reactions to tune relevant properties of the sorbent, such as polarity, hydrophilicity, or surface affinity. ${ }^{109}$ Interestingly, several studies have reported in the possibility of modifying the surface of vinyl-functionalized MWCNTs by incorporation of MIPs synthesized insitu. The approach have been proved to be effective for the extraction of analytes such as chlorphiriphos ${ }^{110}$ or erythromycin ${ }^{111}$ from relatively complex extracts with minimum sorbent demands and solvent consumption.

\subsection{Micro-extraction by packed sorbent}

Micro-extraction by packed sorbent (MEPS) is a modification of SPE in which a small amount of sorbent packing material (ca. $1 \mathrm{mg}$ ) is placed at the top of a syringe needle and used to concentrate analytes by successively withdrawing and ejecting the investigated aqueous matrix 
(typically, 10-250 $\mu \mathrm{L}$ ). Because of the small volume of solvent used for analyte desoption, the technique is suitable for hyphenation with GC, LC and CE. Sorbent materials for MEPS are similar to those used for conventional SPE, although special phases like tailor-made materials (e.g., MIPs) ${ }^{112,113}$ and carbon-based engineered nanomaterials ${ }^{114,115}$ can be particularly suitable for this approach. Depending of the complexity of the matrix investigated and on its (possible) pretreatment before MEPS, reusing of MEPS fibers up to 100 times can be possible. ${ }^{112}$ MEPS can be performed either manually ${ }^{113}$ or using robotic and/or automatic platforms, ${ }^{116}$ which contributes to increase not only sample throughput, but the performance and accuracy of the sample preparation process.

Since its introduction in 2004, the technique has been used for the determination, for instances, of micropollutants, personal care products and pharmaceuticals of divergent nature in environmental waters, biological matrices and foodstuffs. ${ }^{113,117-119}$ However, as for other sorbentbased techniques, MEPS of solid samples is only possible after extraction of the target analytes from the investigated matrix. In addition, depending on the packing sorbent used, previous solvent exchange can be mandatory. In other words, in these types of applications, MEPS is used as a fast preconcentration technique rather than as a pure extraction procedure. The high complexity of some of these extracts may also make advisable the use of highly selective packing materials that contribute to enhance the specificity to the process, such as MIPs ${ }^{113}$ or IL-modified sorbents. ${ }^{120}$ To our knowledge, no study reporting on the feasibility of DES-modified sorbents on the MEPS format can be found in the literature up to now.

\subsection{Miniaturized dispersive solid-phase extraction}

As evidenced in previous sections, the development of new materials for SPE with improved loading capabilities have contributed to significantly reduce of amount of sorbent require for many applications. This has made possible the scaling-down of the dispersive solid-phase extraction ( $d$ SPE) process yielding a miniaturized version of this technique. Miniaturized d-SPE relies on the improved sorption capacity provided by some of the recently introduced sorbents, in particular, nanomaterials. Among them, MWCNTs are frequently preferred as dispersive sorbent because of their large active area and amazing absorption capacity. However, the high affinity of this type of sorbent for compounds of very different nature has frequently made necessary the use of a cosorbent(s) for simultaneous clean-up. This aspect becomes particularly relevant and evident when dealing with the analysis of trace analytes in relatively complex matrices, although its intensity can 
vary sharply depending on the nature of the target compound. Hou et al. ${ }^{121}$ compared the dynamic linear range and scopes of solution and matrix-matched calibration lines prepared from tea extracts subjected to d-SPE with $6 \mathrm{mg}$ of MWCNTs and $150 \mathrm{mg}$ of PSA for simultaneous extraction and purification of selected pesticides. The authors concluded that, while matrix effect was negligible for some pesticides, for others the use of matrix-matched calibration was highly advisable. In any case, the use of PSA as co-sorbent was mandatory in all cases.

As an alternative, surface modified materials can be prepared for improved selectivity. In this field, the use of magnetic nanoparticles (NPs) represents a particularly attractive alternative as these materials can easily retrieved from the extraction solution by applying an external magnetic field so avoiding the laborious and time consuming centrifugation or filtering step required for sorbent isolation after d-SPE. ${ }^{122,123}$ As an illustration of the typical research carried out in this fields and of the potential of the approach, Deng et al. ${ }^{122}$ have recently reported on the efficiency of aminefunctionalized magnetic NPs and MWCNTs (MNPs/MWCNTs) composites for the fast preconcentration and clean-up of pesticides also from tea extracts. Amine functionalized magnetic NPs exhibited weak anion exchange property and, consequently, they may interact strongly with various polar organic acids. Meanwhile, MWCNTs are able to retain large amounts of pigments and sterols. Composites with a MWCNTs:MNPs ratio of 3:7 were found to provide adequate cleanup of the tea extracts and appropriate pesticide recoveries (in the 73-103\% range) and repeatabilities (RSDs lower than 13\%), with LOQs below $0.08 \mathrm{mg} \mathrm{kg}^{-1}$ when using GC-MS for final determination. In another application example, graphene was chemically immobilized on the surface of silica-coated $\mathrm{Fe}_{3} \mathrm{O}_{4}$ nanocomposite and used for enrichment of carbamates from cucumber and pear. In this case, d-SPE was done after minimum sample treatment (viz, centrifugation and filtration of the supernatant) and LODs below $0.2 \mathrm{ng} \mathrm{g}^{-1}$ were obtained using LCUV/Vis. ${ }^{124}$ Other examples involving the modification of NPs with ILs can also been found in the recent literature. ${ }^{125}$

Improved specificity can be achieved by the synthesis of molecularly-imprinted MWCNTs. This approach has been used by Zang et al., ${ }^{126}$ who reported on the use of magnetic NPs of $\mathrm{Fe}_{3} \mathrm{O}_{4}$ coated with molecularly-imprinted MWCNTs for the determination of bovine serum albumin (BSA) in buffered solutions and bovine serum samples. Sorbent characterization by scanning electron microscopy and Fourier transform infrared spectroscopy suggested that MIPs were successfully immobilized on the surfaces of the MWCNTs and that MIPs were located close to the sorbent surface (Figure 3). A small amount of this sorbent, $10 \mathrm{mg}$, sufficed for quantitative and specific 
recovery (92-97\%; RSD, below 4\%) of BSA from $10 \mathrm{~mL}$ of the aqueous sample. The maximum capacity of the sorbent was estimated to be $52.8 \mathrm{mg} \mathrm{g}^{-1}$, and the time required to reach equilibrium at ambient temperature $40 \mathrm{~min}$. Once the extraction step was completed, the sorbent was easily retrieved by the application of a magnetic field and washed with $\mathrm{Tris}-\mathrm{HCl}$ buffer solution (10.0 mmol L $\mathrm{L}^{-1}, \mathrm{pH} 7.0$ ) containing $3.0 \mathrm{mmol} \mathrm{L}^{-1} \mathrm{NaCl}$ to remove the non-specifically adsorbed protein. The specifically adsorbed protein was subsequently recovered with $5.0 \mathrm{mmol} \mathrm{L}^{-1} \mathrm{NaCl}$.

Again, due to the relatively recent introduction of DESs, only a few examples on the performance of DES-modified sorbents in the d-SPE format can be found in the literature. In our opinion, Ghorbanian's group proposed one of the most interesting approaches, which has been evaluated for the determination of traces of nitrosaromatic explosives ${ }^{127}$ and organochlorine pesticides ${ }^{128}$ in aqueous samples. In these studies, the authors prepared a colloidal gel of magnetic carbon nanotube and DES that was used for d-SPE of the target compound. In this gel, the DES, which was compatible with GC, acted simultaneously as both carrier and stabilizer for the magnetic nanotubes, which allowed their easy and fast dispersion on the bulk sample without any extra shaking of the mixture. The impressive enrichment factors achieved (roughly in the 250-400 range) and low LODs (in the $\mathrm{ng} \mathrm{L}^{-1}$ range) demonstrated the feasibility of the optimized methodologies for the intended determination.

An alternative format for miniaturized d-SPE consisted on the loosely packing of a small amount of an appropriate sorbent on a disposable pipette tip in between two frits. The liquid sample (or extract) is aspirated into the tip and the sorbent-analyte interaction improved by air bubbling turbulence. After a preselected extraction time of typically less than $1 \mathrm{~min}$, the liquid phase is ejected and, if required, the process repeated. Then, a small volume of the extraction solvent is aspirated and the target analytes eluted out of the column. The method is fast, simple, easy to automate, full-fills the principles of green chemistry (viz, minimum reagent and energy consumption, and reduced waste generation) and, due to its features, it is particularly suitable for the treatment small-size samples. The technique, so-called disposable pipette extraction (DPX), was initially assayed for the preconcentration of drugs from biological fluids, ${ }^{129}$ and of pesticides from fruits and vegetables extracts. ${ }^{130}$ However, current applications include, for example, the rapid multiresidual analysis of pesticides ${ }^{131}$ and explosives residues, ${ }^{132}$ or the purification of complex biological extracts during the analysis of $\mathrm{PCBs}{ }^{133}$ and antibiotics. ${ }^{134}$ 
The possibility of preparing a home-made 36-syringe d-SPE array by packing of the sorbent in a syringe barrel has recently been investigated by Zhu et al. ${ }^{135}$ The system was applied to the fast extraction of endogenous cytokinins from $O$. sativa. The large surface area of the mesoporous silica fibers used as sorbent allowed quantitative recovery of the target compounds with only 15 $\mathrm{mg}$ of sorbent. This sorbent format simultaneously contributed to increase solvent flow-rates due to the low back-pressure inside the syringe. Efficient sorbent-sample contact and fast mass transfer was ensured by vortexing of the mixture in the syringe. Under optimized conditions, sample extraction of the analytes from the plant extracts was completed in only 4 min and recoveries in the $77-107 \%$ range were reported. The batch-to-batch reproducibility was satisfactory, with RSDs below $13 \%$. All together would demonstrate the potential of this type of sorbent for miniaturized, high throughput, and fast sample preparation on array platforms.

\subsection{Solid-phase microextraction}

SPME was introduced as a miniaturized (virtually) solvent-free technique for preconcentration and/or purification of analytes from gaseous, liquid, viscous and solid samples. In its most popular, widely-used format, SPME consists of a fused silica or metal-wire support coated with an appropriate sorbent layer into which the analyte(s) is(are) adsorbed by simple exposure of the fiber for a preselected time to the head-space above the sample (HS-SPME), or alternatively by direct immersion into the investigated aqueous sample (DI-SPME). SPME is an equilibrium (i.e., non-exhaustive) technique and, similarly to other non-exhaustive techniques previously revised, analytical strategies such as stirring, heating up or derivatization of the sample are common practices to speed up the analyte transfer from the matrix to the SPME fiber coating. Rapid acceptation of the technique as a simple, green, reproducible, and miniaturized methodology that could be applied to the analysis of analytes of widely divergent nature contributed to expand its application to different research areas. Today, full automation of the SPME process can be achieved through a number of systems (e.g., autosamplers) and different platforms allow on-line coupling of SPME with GC-MS ${ }^{136}$ and LC, the latter primarily through the in-tube SPME configuration. ${ }^{137,138}$ For high throughput analysis, novel SPME multi-well-plate-based formats has been introduced in recent years. ${ }^{139}$ Improved features of the multi-well-plate formats over conventional automatic fiber and in-tube SPME include reduced solvent consumption, low cost, reusability, improved selectivity, and compatibility with small-volume samples. ${ }^{140}$ The original

automated rod-based 96 -well-plate system ${ }^{141}$ evolved rapidly to a thin-film configuration that it is 
now commercialized as the Concept 96 SPME robotic sample preparation system. ${ }^{142,143}$ Compared to the rod-based format, the novel blade format used for SPME in thin-film microextraction (TFME) contributed to increase the surface area of the solid support exposed to the sample. This resulted in an improved mass transfer due to the more favorable extractive phase volume (ca. 3.5folds compared to the original rod-based design). In addition, blades allowed a more effective sample agitation. As a consequence, the technique provided improved sensitivity without sacrifying time. TFME format also simplified direct coupling with other instrumental techniques, in particular, with MS. ${ }^{136,144}$ On the other hand, complete immersion of the coating into the sample obliged to increase the minimum sample volume up to $0.8-1.8 \mathrm{~mL}$, and the same consideration applies for the amount of solvent required for analyte desorption. In other words, the practicality of the technique for handling size-limited samples becomes somehow compromised. ${ }^{140}$ Thereby, for this type of applications, the in-tip SPME format can be considered a more adequate format. This configuration consists of a SPME fiber positioned inside a disposable pipette tip and kept in place with a polyethylene frit, although for enhanced extraction efficiency monolithic phases prepared in situ can also be used. The former approach is named fiber-packed in-tip SPME, ${ }^{145}$ while the latter is known as pipette-tip SPME. ${ }^{146,147}$ In both cases, sorbent conditioning and sample preconcentration and desorption is achieved by successive aspiration and ejection cycles in a manner similar to in-tube SPME. Consequently, although adequate for the handling of small-size samples (0.1 $\mathrm{mL}$ sufficed for some applications), its format prevent from application to the treatment of relatively complex matrices. The technique, including its 96 -plate format, can be fully automated using conventional automation systems existing in most laboratories for liquid handling which, in principle, should facilitate its setting-up and adoption. As in pipette-tip SPME, the aspiration speed do not affect the precision of the method, ${ }^{145}$ and complete sample preparation times in the 2-6 min range have been reported for 96 samples using monolithic sorbents. ${ }^{148}$ For in-deep discussion of the principles and relative merits of these two approached for high-throughput analysis, including in-vivo applications and devices, as well as emerging application fields, the reader is referred to reviews of a more specific nature. ${ }^{149-152}$

Similarly to that observed for other sorbent-based techniques, during the last years, SPME has increasingly turned to the refinement of the analyte retention modes through the development of new coatings materials. Achievements in this field have simultaneously contributed to solve some of the most pressing shortcomings of conventional sorbents and to expand the scope and type of application studies afforded with SPME. ${ }^{153}$ The development of biocompatible materials ${ }^{154,155}$ 
allowing in-vivo studies through the design of a disposable device with a hypodermic needle housing the fiber, ${ }^{156}$ or of disk thin films for in vivo saliva sampling ${ }^{157}$ are considered remarkable and illustrative examples in this sense. ${ }^{152}$

Today, apart from the originally introduced non-polar polydimethyl siloxane (PDMS), semi-polar polydimethyl siloxane-divinylbenzene (PDMS-DVB), polar polyacrylate (PA), Carbowaxdivinylbenzene (CW-DVB) liquid-like phases, coated porous particle phases such a polydimethyl siloxane-Carboxen (PDMS-Carboxen), poly(3-methylthiophene) and Nafion are commercialized. Conductible polymers, in particular polyaniline- (PANI), polypyrrol- (PPy) and polythiophene- (PT) based phases, have been proved to be effective alternatives for the preconcentration of volatile, polar and ionic compounds from aqueous matrices. ${ }^{158}$ Nevertheless, despite the (in many occasions) tailored properties of these fibers, their use remains somehow limited. In recent years, the possibility of improving the analytical performance and thermal stability of these coatings by the incorporation of nanomaterials to their preparation has been evaluated. Mehdinia and Mousavi ${ }^{159}$ prepared a nano-structured PANI coating for the first time in 2008. Results revealed that this SPME coating showed higher extraction efficiency for PCBs than the micro-structured PANI coating due to its nanostructure. When this material was electrodeposited in microemulsions containing $[\mathrm{BMIM}]\left[\mathrm{PF}_{6}\right]$, a composite SPME fiber with excellent preconcentration capability for organochlorine pesticides (OCPs) from the HS of the investigated aqueous samples was obtained. ${ }^{160}$ The fiber was cheap and easily to prepare (reproducibility between fibers as RSD was below $11 \%$ ), exhibited a high thermal stability (up to $350^{\circ} \mathrm{C}$ ) and, according to the authors, it could be reutilized for more than 250 times without any obvious decrease of the extraction efficiency. For this particular application, its analytical performance was superior to that provided by conventional PANI and PDMS SPME fibers. Similar advantageous features were observed when PANI was modified with MWCNTs. ${ }^{161}$ In this case, the electrodeposited coating film had a porous structure with higher specific surface area, and enhanced adsorption capacity than the PANI fiber, as illustrated for the HS-SPME of phenolic compounds from aqueous samples (recoveries in the 87-112\% range; RSDs below 7\%; fiber-to-fiber RSD, lower than 12\%). The chemical binding between the Pt substrate and the coating, and the interaction of PANI with the CNTs resulted in a material with high thermal stability (up to $320^{\circ} \mathrm{C}$ ) and excellent re-usability (each fiber could be used more than 250 times). Essentially similar conclusions were drawn from a close-related application study involving a PPy-grafene composite fiber and phenolic compounds as target analytes. ${ }^{162}$ In this case, the coating thickness $(20-30 \mu \mathrm{m})$ was controlled through the 
polymerization time. The extraction efficiency of the PPy-graphene coated fiber was superior to those of CAR/PDMS, PA, PPy, and PPy-graphene oxide. Results reported up to now proved the potential of unexpensive non-covalent procedures for the preparation of thermally stable, homogeneous and high capacity graphene-based SPME coatings. Nevertheless, the improved mechanical stability conferred to the fiber by convalent bonding methods may envisage an increase in research in this field in future.

Siliceous nanoparticles and CNPs are receiving increasing attention as SPME fiber coatings due to their large specific surface area and high adsorption capacity. Different procedures can be used for their physical and chemical immobilization on the supporting fiber surface ${ }^{163}$ and, although efficient extraction properties have been reported for the unmodified material, their chemical modification frequently resulted in improved adsorption capability or selectivity. ${ }^{164,}{ }^{165}$ This becomes particularly true when MIPs are immobilized on the surface of the fiber coating. ${ }^{92}$ Liu et al. ${ }^{166}$ reported on the benefices derived from combining the high selectivity associated to the use of MIP as shape-selective recognition element with the improved extraction efficiency achieved by applying an electrochemically controlled SPME to the extraction of ionic compounds, such as fluoroquinolone antibiotics, from urine and soil extracts. In this study, the authors used a molecularly imprinted polypyrrole/MWCNTs composite coating deposited onto a Pt wire. The application of a current potential to the MIP-PPy/MWCNTs/Pt fiber, which was used as working electrode in a standard three-electrode system, promoted the electrophoretic transfer of fluoroquinolones to the coating surface, from which they entered shape-complimentary MIP cavities by hydrogen-bonding and ion-exchange interactions. The preconcentrated analytes were subsequently desorbed by elution with $400 \mu \mathrm{L}$ of a methanol:acetic acid (80:2, v/v) solution and the enriched solvent analysed by LC-UV. Despite the satisfactory performance and selectivity of the SPME process (recoveries, $85-94 \%$ for the urine samples, and $90-96 \%$ for the soil extracts; average RSDs, below 7\%; and LODs lower than $2 \mu \mathrm{g} \mathrm{L}^{-1}$ ), it should be mentioned that the preconcentration step length for $60 \mathrm{~min}$.

As for SPE, when ILs are immobilized onto the surface of a SPME fiber, they lose their liquid nature, but provide the modify sorbent with a number of properties that have already been exploited in a number application studies and highlighted in several review papers. ${ }^{9,}{ }^{153}$ Physical sorption of IL onto a SPME fiber resulted in relatively weak interactions that negatively affected coating integrity and extraction capacity in direct immersion applications and also limited the possibility of fiber reutilization. The enhanced stability achieved by chemically bonded IL-based 
fibers contributed to solve these shortcomings, as demonstrated in the pioneer study by Amini et al. ${ }^{167}$ In that study, the performance of a newly synthesized IL, 1-methyl-3-(3-trimethoxysilyl propyl) imidazolium bis(trifluoromethylsulfonyl) imide, cross linked to the surface of the fusedsilica fiber, was compared with that of the equivalent physically coated fiber during the HS-SPME of methyl tert-butyl ether (MTBE) from gasoline. The chemically IL-modified fibers showed improved thermal stability (working temperature up to $220^{\circ} \mathrm{C}$ vs $180^{\circ} \mathrm{C}$ in the physically coated fiber), reproducibility (RSD, $9 \%$ vs $12 \% ; n=6)$, and could be reutilized up to 16 times while physically coated fiber reutilization was not possible. IL-based fibers with enhanced thermal and chemical stability have been prepared by using the sol-gel methodology. ${ }^{168}$ Improved selectivity and stability was also achieved by developing functionalized ILs. Although this approach can certainly complicate the synthesis process, ${ }^{169}$ it has also been demonstrated to be a valuable alternative that can contribute to expand the applicability of IL-based fibers. ${ }^{153}$

Despite the improved features of some of these materials, the remaining limitations made the synthesis of so-called polymeric IL (PIL)-based coatings to be considered at present as one of the most valuable alternatives to prepare IL-based fibers with improved thermal and chemical stability, reproducible films and lifetimes comparable to those of conventional SPME films. ${ }^{9}$ PILs are synthesized from IL monomers and exhibit intrinsic polymer characteristics while retaining the tunable chemical features of ILs. In general, they are structurally polyelectrolites insoluble in water, which made them feasible for direct immersion SPME. In a series of illustrative examples, Anderson's group ${ }^{170}$ proposed the use of the PIL poly(1-vinyl-3-hexadecylimidazolium) bis[(trifluoromethyl)sulfonyl]imide (poly([VHDIM] [NTf $\left.{ }_{2}\right]$ ) as a novel SPME fiber coating material for the DI-SPME of eighteen pollutants, including polycyclic aromatic hydrocarbons (PAHs) and substituted phenols, from waters with satisfactory results. Functionalization of the PIL with benzyl groups to yield poly(1-4-vinylbenzyl)-3-hexadecylimidazolium bis[(trifluoromethyl)sulfonyl]imide (poly([VBHDIM][NTf $]$ ) enhanced $\pi-\pi$ interactions between the sorbent coating and the target analytes. This resulted in an impressive selectivity towards the extraction of particular PAHs compared to the not functionalized PIL [poly(1-vinyl-3-hexadecylimidazolium bis[(trifluoromethyl)sulfonyl]imide), poly[HDIM][NTf 2$]]$, and substantially lower LODs: 0.003-0.07

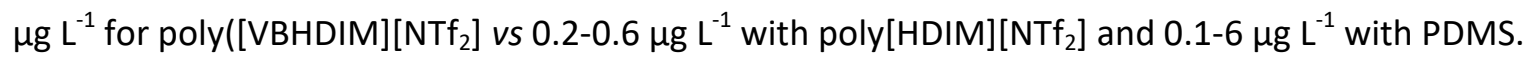
Replacing of the hydrophobic counter-anion used in these PILs by $\mathrm{Cl}^{-}$, as in poly(1-vinyl-3hexylimidazolium chloride) (poly[VHIM][Cl]), reduced the coating solubility in organic solvents and improved their affinity for polar compounds. ${ }^{171}$ Unfortunately, this simultaneously reduced their 
thermal stability of the fiber so compromising the possibility of reutilization. Preparation of chemically-bonded PILs has also been investigated. ${ }^{172}$ Despite the promising results, the fabrication procedures of this type of fiber should still be improved to increase fiber-to-fiber reproducibility and their average lifetime.

In-deep discussion on the advantages, remaining shortcoming and future perspectives on the use of these and other sorbents can be found in recent review papers. ${ }^{105,153,173}$

\subsection{Stir-bar sorptive extraction}

Stir-bar sorptive extraction (SBSE) is another interesting, miniaturized, simple and environmentally friendly extraction technique. In its most common format, a magnetic stirring rod contained in a glass jacket covered with an appropriated sorbent is directly immersed in the investigated liquid sample or matrix extract for analyte preconcentration for a preselected (in general, fairly long) time. Afterwards, the stir-bar is retrieved from the sample, dried and the analytes desorbed from the enriched sorbent phase by either thermal desorption in the injection port of the GC, or by elution with a (small) volume of an appropriate solvent for subsequent separation-plus-detection. The SBSE rod can also be exposed to the HS of a vial containing a gaseous, liquid or solid sample, but this approach is much less common. The amount of phase involved in SBSE is ca. 100-fold larger than that used in conventional SPME. Hence, an improved phase ratio is achieved, which yields improved extraction efficiency and lower LODs compared to SPME. At present, SBSE is considered a well-stablished and accepted technique in application fields such as environmental and food analysis and, in less extension, pharmaceutical and clinical research $\left({ }^{174}\right.$ and references therein).

Irrespective of the SBSE format, for years, the main shortcomings of the technique were the limited number of commercially available coatings (initially restricted to PDMS), and the difficulty of full automation. ${ }^{175}$ The latter remains as a handicap for the technique. However, efforts made in the former field resulted in the development of, primarily, dual-phase/hybrid twisters, in which the conventional PDME phase was combined with another sorbent to increase the selectivity and/or efficiency of the extraction process. ${ }^{176}$ Later on, novel coating materials with improved analytical features were introduced, ${ }^{177,}{ }^{178}$ although not all of them are commercially available. ${ }^{174}$ Alternatively, novel working modes for simultaneous extraction of analytes with different polarity, such as multishot, ${ }^{179}$ sequential extraction, ${ }^{180}$ ice concentration linked with extractive stirrer

(ICECLES), ${ }^{181,182}$ or solvent-assisted SBSE; ${ }^{183}$ and new SBSE configurations, such as dual-solvent 
SBSE, in which the organic phase was confined to a pair of hollow-fiber membranes fixed on a stir bar, ${ }^{184}$ has been investigated. The feasibility of SBSE with in-situ and in-tube derivatización, as well as in-situ deconjugation has also been demonstrated and discussed in different recent review articles. ${ }^{178,185,} 186$ Despite the improved extraction efficiency provided for some of these approaches compared to conventional SBSE (see Figure 4 for a typical example involving SASBSE), ${ }^{186}$ final acceptation of these new SBSE-based working modes for general application studies looks to be rather different. ${ }^{174}$

Apart from this, a variety of novel coating materials have been assayed as SBSE sorbents during the last decade. Among then, monolitic materials have demonstrated to be particularly suitable for SBSE. Apart from the simplicity and low cost of theit preparation, these sorbents show high permeability, which favors mass-transfer and contributes to reduce the extraction time. In a recent study, ${ }^{187}$ the potential of a novel polar monolithic coating, poly(PEGMA-Co-PETRA), obtained by copolymerization of monovinyl monomer poly(ethylene glycol) monomethacrylate (PEGMA) with pentaerythritol triacrylate (PETRA) as cross-linking agent, was evaluated for the simultaneous extraction of a group of polar and non-polar emerging microcontaminants from synthetic water. The promising results reported encourage research in this field and further evaluation by application to the analysis of more complex matrices. The application of sol-gel technology to the preparation of SBSE coatings resulted in phases with improved thermal and chemical stability, low bleeding, and relatively long lifetime due to the strong adhesion achieved between the coating and the glass surface. ${ }^{185}$ The facility to introduce chemical groups with different functionally during the preparation of the coating material is an additional advantage associated to this methodology. A novel IL-bonded sol-gel stir bar coating was prepared by chemically binding an n-vinyl imidazolium-based ILs, $[\mathrm{AIM}]\left[\mathrm{BF}_{4}\right]$, to the surface of bare stir bar with KH-570 as bridging agent. The material showed a satisfactory mechanical strength and durability and provided recoveries in the $81-116 \%$ range for nonsteroidal anti-inflammatory drugs (i.e., Ketoprofen, naproxen and fenbufen) after $30 \mathrm{~min}$ of SBSE from the test water. ${ }^{188}$ Application to more complex liquid or viscous matrices required previous sample pretreatment, such as dilution and filtration in the case of urine, or defatting and protein precipitation for milk. For these types of application studies, coatings with more selective recognition capabilities, as those based on biocompatible RAM ${ }^{189}$ or, even better, MIP ${ }^{92,190}$ coatings, should be preferred. 
As already shown for other sorbent-based techniques, SBSE has also been beneficed by advances achieved in the nanomaterials science. As a representative example, it can be mentioned the development of a poly(ethylene glycol dimethacrylate)-graphene composite as SBSE coating. ${ }^{191}$ This new material was used for the preconcentration of PAHs from aqueous samples. Compared to the neat polymer, the graphene-polymer composite material showed a much higher specific surface area (i.e., 4.4-folds increase) and improved affinity for the test compounds. In another study, $^{192}$ an amino modified MWCNTs/polydimethylsiloxane (MWCNTs-4,4'diaminodiphenylmethane/polydimethylsiloxane, MWCNTs-DDM/PDMS) was synthesized, and utilized for the SBSE of phenols from environmental water and soil samples. Under optimized conditions, the proposed method showed a liner response over three ranges of magnitude, adequate LODs in the $0.1-1.8 \mu \mathrm{g} \mathrm{L}^{-1}$ range, and provided enrichments factors as large as 63 for some of the target compounds.

Modified magnetic NPs have also been evaluated in the preparation of SBSE coating with improved features. In contracts to their non-magnetic homologues, magnetic NPs can move (and so be efficiently isolated) by application of an external magnetic field. In a recent study, lipophilic oleic acid-coated cobalt ferrite $\left(\mathrm{CoFe}_{2} \mathrm{O}_{4} @\right.$ oleicacid) magnetic NPs were used as hydrophobic coating physically supported on a neodymium-core stirring bar to yield a SBSE modification that combined the principles of this techniques with those of $d$-SPE. This novel analytical was named stir bar sorptive-dispersive microextraction (SBSDE). ${ }^{173,}{ }^{193}$ In a typical experiment, the coated magnetic stir bar was immersed into a sample solution. As long as a relatively slow stirring rate was applied, magnetism was strong enough to retain the modified magnetic NPs attached to the bar surface. Under these conditions, the extraction was performed in a similar manner as SBSE. As stirring rate increased, centrifugal forces increased and, at a certain point, the NPs were dispersed into the aqueous solution and extraction was afforded alike d-SPE. Once the extraction time was completed, the stirring process finished. At this point, the strong magnetic field of the stir bar prevailed again and the dispersed magnetic NPs were rapidly retrieved. Then, the stir bar was collected and the preconcentrated analytes were back-extracted into an appropriate solvent for subsequent chromatographic separation and detection. The performance of this novel SBSE-based approach was illustrated by successful application to the analysis of eight common UV-filters in seawater. The method showed good analytical features in terms of linearity, enrichment factors (11-148), LODs (low $\mu \mathrm{g} \mathrm{L}^{-1}$ ), intra- and inter-day repeatability (RSD $\left.<11 \%\right)$, and relative recoveries (87-120\%). More importantly, it demonstrated the practical potential of this new and suitable 
approach that can easily adapted for application in other studies, so contributing to expand the potential of SBSE in near future.

\section{Conclusions}

Progress done during the last decades in the field of sample preparation has yielded a number of novel, and frequently miniaturized, techniques that have contributed to green this part of the analytical process. These techniques have contributed to solve, at least partially, some of the most pressing shortcomings of conventional (i.e., large-scale) sample treatment procedures, viz. consumption of large amounts of sample, reagents and energy, slow analytical response time, and wastes generation. Today, sample preparation can be completed with minimum sample manipulation, in a short time and, in many instances, in an unattended and/or hyphenated manner, especially in the case of aqueous matrices. Novel sample preparation techniques are also better suited for the treatment of size-limited samples and, when combined with an appropriated instrumental system, proper analyte detection and quantitation is possible even at the low-trace level. In recent years, these novel techniques have greatly benefited from advances achieved in other research areas, especially those related to the development of new materials and nanotechnologies. Investigation on the feasibility of new safe and non-toxic extraction media with improved analytical features, with IL, DES, and supramolecular solvents as representative examples, is a recognizable trend in this field that effectively contributes to the greening of the sample preparation procedures. However, as demonstrated in this text, despite their many positive analytical features of most of these novel analytical approaches and techniques, problems remain, so limiting the general acceptation and wide use of some of these approaches and techniques; but also stimulating further investigation in active research area.

\section{Acknowledgement}

Author thanks MICINN, Comunidad of Madrid and European funding from FSE and FEDER for projects AGL2016-80475-R and S2018/BAA-4393 (AVANSECAL-II-CM).

\section{References}

1. S. Armenta, S. Garrigues and M. de la Guardia, TRAC Trends Anal. Chem., 2015, 71, 2-8. 
2. L. Ramos, J. Chromatogr. A, 2012, 1221, 84- 98.

3. M. Pena-Abaurrea and L. Ramos, in RSC Green Chemistry Series, eds. M. de la Guardia and S. Garrigues, RSC Publishing, Cambridge (UK), 2011, pp. 107-143.

4. L. Ramos and B. Richter, LC-GC Europe, 2016, 29, 558-568.

5. C. Ribeiro, A. R. Ribeiro, A. S. Maia, V. M. F. Goncalves and M. E. Tiritan, Crit. Rev. Anal. Chem., 2014, 44, 142-185.

6. A. Spietelun, L. Marcinkowski, M. de la Guardia and J. Namieśnik, Talanta, 2014, 119, 3445.

7. Q. Liu, Q. Zhou and G. Jiang, Trends Anal. Chem., 2014, 58, 10-22.

8. A. Samsidar, S. Siddiquee and S. M. Shaarani, Trends Food Sci. Technol., 2018, 71, 188-201.

9. L. Ruiz-Aceituno, M. L. Sanz and L. Ramos, TRAC Trends Anal. Chem., 2013, 43, 121-145.

10. M. Espino, M. D. Fernandez, F. J. V. Gomez and M. F. Silva, TRAC Trends Anal. Chem., 2016, 76, 126-136.

11. H. Vanda, Y. T. Dai, E. G. Wilson, R. Verpoorte and Y. H. Choi, C. R. Chim., 2018, 21, 628638.

12. W. Liu, K. D. Zhang, G. L. Yang and J. J. Yu, Food Chem., 2019, 281, 140-146.

13. T. Khezeli, A. Daneshfar and R. Sahraei, Talanta, 2016, 150, 577-585.

14. Y. Yamini, M. Rezazadeh and S. Seidi, TRAC Trends Anal. Chem., 2019, 112, 264-272.

15. N. Campillo, P. Vinas, J. Sandrejova and V. Andruch, Appl. Spectrosc. Rev., 2017, 52, 267415.

16. H. Ebrahimzadeh, F. Mirbabaei, A. A. Asgharinezhad, N. Shekari and N. Mollazadeh, J. Chromatogr. B, 2014, 947-948, 75-82.

17. J. M. Kokosa, A. Przyjazny and M. A. Jennot, Solvent microextraction. Theory and practice, Wiley, New Jersey (USA), 2009.

18. S. Tang, T. Qi, P. D. Ansah, J. C. N. Fouemina, W. Shen, C. Basheer and H. K. Lee, TRAC Trends Anal. Chem., 2018, 108, 306-313.

19. M. Saraji and M. K. Boroujeni, Anal. Bioanal. Chem., 2014, 406, 2027-2066.

20. L. Wang, Z. M. Wang, H. H. Zhang, X. Y. Li and H. Q. Zhang, Anal. Chim. Acta, 2009, 647, 7277.

21. D. B. G. Williams, M. J. George, R. Meyer and L. Marjanoyic, Anal. Chem-, 2011, 83, 67136716.

22. H. Bagheri, M. Dehghan, A. Ali Es'haghi and M. Naderi, Anal. Methods, 2013, 5, 4846-4851. 
23. E. Ghasemi, J. Chromatogr. A, 2012, 1251, 48-53.

24. Y. Yaminia, S. Seidib and M. Rezazadeh, Anal. Chim. Acta, 2014, 814, 1-22.

25. A. Melnyk, L. Wolska and J. Namiesnik, J. Chromatogr. A, 2014, 1339, 1-12.

26. A. Shishov, N. Volodina, D. Nechaeva, S. Gagarinova and A. Ulatov, Microchem. J., 2019, $144,23-32$.

27. B. Tang, W. Bi, H. Zhang and K. H. Row, Chromatographia, 2014, 77, 373-377.

28. C. Carrillo-Carrion, B. Simonet and M. Valcarcel, Analyst, 2012, 137, 1152-1159.

29. X. Guo, D. Yin, J. Peng and X. Hu, J. Sep. Sci., 2012, 35, 452-458.

30. C. Ruiz-Palomero, M. L. Soriano and V. M., Talanta, 2014, 125, 72-77.

31. F. J. Lopez-Jimenez, S. Rubio and D. Perez-Bendito, J. Chromatogr. A, 2008, 1195, 25-33.

32. L. Qian and Y. He, J. Chromatogr. A, 2006, 1134, 32-37.

33. Y. Tao, J. F. Liu, X. L. Hu, H. G. Li, T. Wang and G. B. Jiang, J. Chromatogr. A, 2009, 1216, 6259-6266.

34. Y. Zhang, R. Wang, P. Su and Y. Yang, Anal. Methods, 2013, 5, 5074-5078.

35. Y. Liu, E. Zhao, W. Zhu, H. Gao and Z. Zhou, J. Chromatogr. A, 2009, 1216, 885-891.

36. A. Zhao, X. Wang, M. Ma, W. Wang, H. Sun, Z. Yan, Z. Xu and H. Wang, Microchim. Acta, $2012,177,229-236$.

37. S. Gao, X. Yang, W. Yu, Z. Liu and H. Zhang, Talanta, 2012, 99, 875-882.

38. Y. C. Fan, Z. L. Hu, M. L. Chen, C. S. Tu and Y. Zhu, Chin. Chem. Lett., 2008, 19, 985-987.

39. S. Sun, Y. Wang, W. Yu, T. Zhao, S. Gao, M. Kang, Y. Zhang, H. Zhang and Y. Yu, J. Sep. Sci., 2011, 34, 1730-1737.

40. M. A. Farajzadeh, A. S. Hojghan and M. R. A. Mogaddam, J. Food Compost. Anal., 2018, 66, 90-97.

41. F. Aydin, E. Yilmaz and M. Soylak, Food Chem., 2018, 243, 442-447.

42. J. Cheng, Y. Xia, Y. Zhou, F. Guo and G. Chen, Anal. Chim. Acta, 2011, 701, 86-91.

43. Z. H. Yang, P. Wang, W. T. Zhao, Z. Q. Zhou and D. H. Liu, J. Chromatogr. A, 2013, 26, 5863.

44. M. Cruz-Vera, R. Lucena, S. Cárdenas and M. Valcárcel, J. Chromatogr. A, 2009, 1216, 6459-6465.

45. K. Yavir, L. Marcinkowski, R. Marcinkowska, J. Namieśnik and A. Kloskowski, Anal. Chim. Acta, 2019, 1054, 1-16. 
46. J. F. Liu, G. B. Jiang, Y. G. Chi, Y. Q. Cai, Q. X. Zhou and J. T. Hu, Anal. Chem., 2003, 75, 5870-5876.

47. E. Aguilera-Herrador, R. Lucena, S. Cardenas and M. Valcarcel, Anal. Chem., 2008, 80, 793800.

48. A. Gjelstad and S. Pedersen-Bjergaard, Anal. Methods, 2013, 5, 4549-4557.

49. A. Chisvert, I. P. Roman, L. Vidal and A. Canals, J. Chromatogr. A, 2009, 1216, 1290-1295.

50. E. Aguilera-Herrador, R. Lucena, S. Cardenas and M. Valcarcel, J. Chromatogr. A, 2009, 1216, 5580-5587.

51. C. Yao, W. R. Pitner and J. L. Anderson, Anal. Chem., 2009, 81, 5054-5063.

52. Q. Zhang, K. D. Vigier, S. Royer and F. Jerome, Chem. Soc. Rev., 2012, 41, 7108-7146.

53. A. P. Abbott, G. Capper, D. L. Davies, H. L. Munro, R. K. Rasheed and V. Tambyrajah, ChemComm., 19, 2010-2011.

54. S. C. Cunha and J. O. Fernandes, TRAC Trends Anal. Chem., 2018, 105, 225-239.

55. G. Shen and H. K. Lee, Anal. Chem., 2003, 75, 98-103.

56. G. Ouyang, W. Zhao and J. Pawliszyn, J. Chromatogr. A, 2007, 1138, 47-54.

57. S. P. Huang and S. D. Huang, J. Chromatogr. A, 2006, 1135, 6-11.

58. N. J. Petersen, H. Jensen, S. H. Hansen, S. T. Foss, D. Snakenborg and S. PedersenBjergaard, Microfluid Nanofluid, 2010, 9, 881-888.

59. G. Jiang and H. K. Lee, Anal. Chem., 2004, 76, 5591-5596.

60. J. A. Lopez-Lopez, C. Mendiguchia, J. J. Pinto and C. Moreno, TRAC Trends Anal. Chem., $2019,110,57-65$.

61. F. A. Hansen, D. Sticker, J. P. Kutter, N. J. Petersen and S. Pedersen-Bjergaard, Anal. Chem., 2018, 90, 9322-9329.

62. N. J. Petersen, J. S. Pedersen, N. N. Poulsen, H. Jensen, C. Skonberg, S. H. Hansen and S. Pedersen-Bjergaard, Analyst, 2012, 137, 3321-3327.

63. N. J. Petersen, S. T. Foss, H. Jensen, S. H. Hansen, C. Skonberg, D. Snakenborg, J. P. Kutter and S. Pedersen-Bjergaard, Anal. Chem., 2011, 83, 44-51.

64. N. K. Drouin, P., S. Rudaz, S. Pedersen-Bjergaard and J. Schappler, TRAC Trends Anal. Chem., 2019, 113, 357-363.

65. L. Y. Guan, Q. Luo, J. Y. Shi and W. Yu, J. Sep. Sci., 2018, 41, 868-876.

66. M. Rezaee, Y. Assadi, M. R. Milani-Hosseini, E. Aghaee, F. Ahmadi and S. Berijani, J. Chromatogr. A, 2006, 1116, 1-9. 
67. M. A. Farajzadeh, N. Nouri and P. Khorram, Trends Anal. Chem., 2014, 55, 14-23.

68. J. Hu, L. Y. Fu, X. N. Zhao, X. J. Liu, H. L. Wang, X. D. Wang and L. Y. Dai, Anal. Chim. Acta, 2009, 640, 100-105.

69. C. Yang, J. Wang and D. Li, Anal. Chim. Acta, 2013, 799, 8- 22.

70. C. Bosch-Ojeda and F. Sanchez-Rojas, Chromatographia, 2011, 74, 651-679.

71. S. Nojavan, T. Gorji, S. S. H. Davarani and A. Morteza-Najarian, Anal. Chim. Acta, 2014, 838, 51-57.

72. V. Andruch, M. Burdel, L. Kocúrová, J. Šandrejová and I. S. Balogh, Trends Anal. Chem., 2013, 49, 1-19.

73. C. Jia, X. Zhu, J. Wang, E. Zhao, M. He, L. Chen and P. Yu, J. Chromatogr. A, 2010, 1217, 5868-5871.

74. J. Regueiro, M. Llompart, C. Garcia-Jares, J. C. Garcia-Monteagudo and R. Cela, J. Chromatogr. A, 2008, 1190, 27-38.

75. L. Kocúrová, I. S. Balogh, J. Šandrejová and V. Andruch, Microchem. J., 2012, 102, 11-17.

76. H. Sereshti, P. Khorram and N. Nouri, Sep. Purif. Rev., 2019, 48, 159-178.

77. B. Xu, D. Q. Song, Y. P. Wang, Y. Gao, B. C. Cao, H. Q. Zhang and Y. Sun, J. Sep. Sci., 2014, 37, 1967-1973.

78. M. Li, J. H. Zhang, Y. B. Li, B. Peng, W. F. Zhou and H. X. Gao, Talanta, 2013, 107, 81-87.

79. H. Z. Wang, L. Hu, W. Z. Li, X. L. Yang, R. H. Lu, S. B. Zhang, W. F. Zhou, H. X. Gao and J. Li, Talanta, 2017, 162, 625-633.

80. W. Yu, Z. Liu, S. Cui, S. Zhang, X. Yang, L. Lei, H. Zhang and A. Yu, Anal. Methods, 2014, 6, 2545-2552.

81. Z. Shen, Z. He, P. Wang, Z. Zhou, M. Sun, J. Li and D. Liu, Anal. Chim. Acta, 2013, 793, 3743.

82. Y. Li, P. S. Chen and S. D. Huang, J. Chromatogr. A, 2013, 1300, 51-57.

83. M. Rogeberg, H. Malerod, H. Roberg-Larsen, C. Aass and S. R. Wilson, J. Pharm. Biomed. Anal., 2014, 87, 120-129.

84. G. A. K. Khan, R. Lindberg, R. Grabic and J. Fick, J. Pharm. Biomed. Anal., 2012, 66, 24-32.

85. N. Fontanals, P. A. G. Cormack, D. C. Sherrington, R. M. Marcé and F. Borrull, J. Chromatogr. A, 2010, 1217, 2855-2861.

86. E. V. S. Maciel, A. L. de Toffoli and F. M. Lancas, Electrophoresis, 2018, 13, 1582-1596. 
87. S. Valsecchi, S. Polesello, M. Mazzoni, M. Rusconi and M. Petrovic, Trends Environ. Anal. Chem., 2015, 8, 27-37.

88. N. Fontanals, R. M. Marce, F. Borrull and P. A. G. Cormack, Trends Anal. Chem., 2010, 29, 765-779.

89. C. Emotte, O. Heudi, F. Deglave, A. Bonvie, L. Masson, F. Picard, A. Chaturvedi, T. Majumdar, A. Agarwal, R. Woessner and O. Kretz, J. Chromatogr. B, 2012, 895-896, 1-9.

90. M. E. Lame, E. E. Chambers and M. Blatnik, Anal. Biochem., 2011, 419, 133-139.

91. F. Augusto, L. W. Hantao, N. G. S. Mogollon and S. C. G. N. Braga, Trends Anal. Chem., $2013,43,14-23$.

92. A. Martin-Esteban, Trends Anal. Chem., 2013, 45, 169-181.

93. W. W. Wang, S. Q. Liu, Y. Xue, Y. Wang and C. Yan, Chin. J. Chromatogr., 2017, 35, 99-104.

94. T. S. Romig, C. Bell and D. W. Drolet, J. Chromatogr. B, 1999, 731, 275-284.

95. F. Chapuis-Hugon, A. Boisbaudry, B. Madru and V. Pichon, Anal. Bioanal. Chem., 2011, 400, 1199-1207.

96. N. Fontanals, F. Borrull and R. M. Marce, Trends Anal. Chem., 2012, 41, 15-26.

97. L. Vidal, J. Parshintsev, K. Hartonen, A. Canals and M. L. Riekkola, J. Chromatogr. A, 2012, 1226, 2-10.

98. D. Bratkowska, N. Fontanals, S. Ronka, F. Borrull, A. W. Trochimczuk and R. M. Marce, J. Sep. Sci., 2012, 35, 1953-1958.

99. G. Fang, J. Chen, J. Wang, J. He and S. Wang, J. Chromatogr. A, 2010, 1217, 1567-1574.

100. K. R. Gan, W. Y. Tang, T. Zhu, W. Li, H. Y. Wang and X. J. Liu, J. Liq. Chromatogr. Relat. Tech., 2016, 39, 882-888.

101. L. L. Liu, W. Y. Tang, B. K. Tang, D. D. Han, K. H. Row and T. Zhu, J. Sep. Sci., 2017, 40, 18871895.

102. G. Li, W. S. Ahn and K. H. Row, J. Sep. Sci., 2016, 39, 4465-4473.

103. G. Li, T. Zhu and K. H. Row, J. Sep. Sci., 2017, 40, 625-634.

104. S. Gangula, S. Y. Suen and E. D. Conte, Microchem. J., 2010, 95, 2-4.

105. L. N. Xu, X. Y. Qi, X. J. Li, Y. Bai and H. W. Liu, Talanta, 2016, 146, 714-726.

106. H. Bagheri, S. Asgari and H. Piri-Moghadam, Chromatographia, 2014, 77, 723-728.

107. J. H. Kim, E. T. Hwang, K. K. Kang, R. Tatavarty and M. B. Gu, J. Mater. Chem., 2011, 21, 19203-19206.

108. R. Q. Long and R. T. Yang, J. Am. Chem. Soc., 2001, 123, 2058-2059. 
109. J. Tian, J. Xu, F. Zhu, T. Lu, C. Su and G. Ouyang, J. Chromatogr. A 2013, 1300, 2- 16.

110. T. S. Anirudhan and S. Alexander, J. Chem. technol. Biotechnol., 2013, 88, 1847-1858.

111. Z. Zhang, X. Yang, H. Zhang, M. Zhang, L. Luo, Y. Hu and S. Yao, J. Chromatogr. B, 2011, 879, 1617-1624.

112. S. M. Daryanavard, A. Jeppsson-Dadoun, L. I. Andersson, M. Hashemi, A. Colmsjo and M. Abdel-Rehim, Biomed. Chromatogr., 2013, 27, 1481-1488.

113. W. Du, C. Lei, S. Zhang, G. Bai, H. Zhou, M. Sun, Q. Fu and C. Chang, J. Pharm. Biomed. Anal., 2014, 91, 160-168.

114. H. Bagheri, Z. Ayazi, A. Aghakhani and N. Alipour, J. Sep. Sci., 2012, 35, 114-120.

115. A. L. de Toffoli, E. V. S. Maciel, B. H. Fumes and F. M. Lancas, J. Sep. Sci., 2018, 41, 288-302.

116. H. Vlčkováa, J. Janáka, T. Gottvalda, F. Trejtnarb, P. Solicha and L. Novákova, J. Pharm. Biomed. Anal., 2014, 88, 337-344.

117. M. Abdel-Rehima, Anal. Chim. Acta, 2011, 701, 119-128.

118. Y. He and M. Concheiro-Guisan, Biomed. Chromatogr., 2019, 33, e4444

119. L. Yang, R. Said and M. Abdel-Rehim, J. Chromatogr. B, 2017, 1043, 33-43.

120. K. Z. Mousavi, Y. Yamini, B. Karimi, S. Seidi, M. Khorasani, M. Ghaemmaghami and H. Vali, Microchim. Acta, 2019, 186.

121. X. Hou, S. R. Lei, S. T. Qiu, L. A. Guo, S. G. Yi and W. Liu, Food Chem., 2014, 153, 121-129.

122. X. Deng, Q. Guo, X. Chen, T. Xue, H. Wang and P. Yao, Food Chem., 2014, 145, 853-858.

123. X. Zhao, Y. Shi, T. Wang, Y. Cai and G. Jiang, J. Chromatogr. A, 2008, 1188, 140-147.

124. M. Sun, X. X. Ma, J. T. Wang, W. N. Wang, Q. H. Wu, C. Wang and Z. Wang, J. Sep. Sci., 2013, 36, 1478-1485.

125. S. Jamshidi, M. K. Rofouei and G. Thorsen, J. Sep. Sci., 2019, 42, 698-705.

126. Z. Zhang, X. Yang, X. Chen, M. Zhang, L. Luo, M. Peng and S. Yao, Anal. Bioanal. Chem., 2011, 401, 2855-2863.

127. A. R. Zarei, M. Nedaei and S. A. Ghorbanian, J. Mol. Liq., 2017.

128. S. M. Yousefi, F. Shemirani and S. A. Ghorbanian, Talanta, 2017, 168, 73-81.

129. S. T. Ellison, W. E. Brewer and S. L. Morgan, J. Anal. Toxicol., 2009, 33, 356-365.

130. H. X. Guan, W. E. Brewer, S. T. Garris, C. Craft and S. L. Morgan, J. Chromatogr. A, 2010, $1217,1867-1874$

131. P. Kaewsuya, W. E. Brewer, J. Wong and S. L. Morgan, J. Agric. Food Chem., 2013, 61, 2299-2314. 
132. H. Guan and K. Stewart, Anal. Lett., 2014, 47, 1434-1447.

133. M. Pena-Abaurrea, V. S. García de la Torre and L. Ramos, J. Chromatogr. A, 2013, 1317, 223-229.

134. S. J. Lehotay, K. Mastovska, A. R. Lightfield, A. Nunez, T. Dutko, C. Ng and L. Bluhm, J. Chromatogr. A, 1313, 103-112.

135. G. T. Zhu, X. M. He, B. D. Cai, H. Wang, J. Ding, B. F. Yuan and Y. Q. Feng, Analyst, 2014, $139,6266-6271$.

136. J. Deng, Y. Yang, X. Wang and T. Luan, Trends Anal. Chem., 2014, 55, 55-67.

137. H. L. Lord, J. Chromatogr. A, 2007, 1152, 2-13.

138. D. Vuckovic, X. Zhang, E. Cudjoe and J. Pawliszyn, J. Chromatogr. A, 2010, 1217, 40414060.

139. D. Vuckovic, E. Cudjoe, D. Hein and J. Pawliszyn, Anal. Chem., 2008, 80, 6870.

140. D. Vuckovic, Trends Anal. Chem., 2013, 45, 136-153.

141. J. P. Hutchinson, L. Setkova and J. Pawliszyn, J. Chromatogr. A, 2007, 1149, 127-137.

142. E. Cudjoe, D. Vuckovic, D. Hein and J. Pawliszyn, Anal. Chem., 2009, 81, 4226-4232.

143. F. S. Mirnaghi, Y. Chen, L. M. Sidisky and J. Pawliszyn, Anal. Chem., 2011, 83, 6018-6025.

144. F. S. Mirnaghi, D. Hein and J. Pawliszyn, Chromatographia, 2013, 76, 1215-1223.

145. W. Xie, W. M. Mullett, C. Miller-Stein and J. Pawliszyn, J. Chromatogr. B, 2009, 877, 415420.

146. W. Xie, C. Chavez-Eng, W. Fang, M. L. Constanzer, B. K. Matuszewski, W. M. Mullett and J. Pawliszyn, J. Chromatogr. B, 2011, 879, 1457.

147. I. Ueta and Y. Saito, Anal. Sci., 2014, 30, 105-110.

148. J. Luckwell and A. Beal, Bioanalysis, 2011, 3, 1227-1239.

149. G. A. Gomez-Rios, M. Tascon and J. Pawliszyn, Bioanalysis, 2018, 10, 257-271.

150. E. A. Souza-Silva, R. F. Jiang, A. Rodriguez-Lafuente, E. Gionfriddo and J. Pawliszyn, TRAC Trends Anal. Chem., 2015, 71, 224-235.

151. E. A. Souza-Silva, E. Gionfriddo and J. Pawliszyn, TRAC Trends Anal. Chem., 2015, 71, 236248.

152. E. A. Souza-Silva, N. Reyes-Garces, G. A. Gomez-Rios, E. Boyaci, B. Bojko and J. Pawliszyn, TRAC Trends Anal. Chem., 2015, 71, 249-264.

153. H. Piri-Moghadam, M. N. Alam and J. Pawliszyn, Anal. Chim. Acta, 2017, 984, 42-65.

154. F. S. Mirnaghi, M. R. N. Monton and J. Pawliszyn, J. Chromatogr. A, 2011, 1246, 2-8. 
155. F. S. Mirnaghi and J. Pawliszyn, J. Chromatogr. A, 2012, 1261, 91-98.

156. D. Vuckovic, R. Shirey, Y. Chen, L. Sidisky, C. Aurand, K. Stenerson and J. Pawliszyn, Anal. Chim. Acta, 2009, 638, 175-185.

157. R. Jiang and J. Pawliszyn, Trends Anal. Chem., 2012, 39, 245-253.

158. H. Bagheri, Z. Ayazi and M. Naderi, Anal. Chim. Acta, 2013, 767, 1-13.

159. A. Mehdinia and M. F. Mousavi, J. Sep. Sci., 2008, 31, 3565-3572.

160. Z. Gao, W. Li, B. Liu, F. Liang, H. He, S. Yang and C. Sun, J. Chromatogr. A, 2011, 1218, 6285-6291.

161. W. Du, F. Zhao and B. Zeng, J. Chromatogr. A, 2009, 1216, 3751-3757.

162. J. Zou, X. Song, J. J., W. Xu, J. Chen, J. Y., W. Y. and X. Chen, J. Sep. Sci., 2011, 34, 27652772.

163. A. Kabir, K. G. Furton and A. Malik, Trends Anal. Chem., 2013, 45, 197-218.

164. H. Bagheri and A. Roostaie, J. Chromatogr. A, 2012, 1238, 22-29.

165. Y. Sun, W. Y. Zhang, J. Xing and C. M. Wang, Microchim. Acta, 2011, 173, 223-229.

166. X. Liu, X. Wang, F. Tan, H. Zhao, X. Quan, J. Chen and L. Li, Anal. Chim. Acta, 2012, 727, 2633.

167. R. Amini, A. Rouhollahi, M. Adibi and A. Mehdinia, J. chromatogr. A, 2011, 1218, 130-136.

168. M. Liu, X. Zhou, Y. Chen, H. L. Liu, X. Feng, G. Qiu, F. Liu and Z. Zeng, Anal. Chim. Acta, 2010, 683, 96-106.

169. X. Zhou, P. F. Xie, J. Wang, B. B. Zhang, M. M. Liu, H. L. Liu and X. H. Feng, J. Chromatogr. A, 2011, 1218, 3571-3580.

170. J. López-Darias, V. Pino, J. L. Anderson, C. M. Graham and A. M. Afonso, J. Chromatogr. A, $2010,1217,1236-1243$.

171. Y. Meng, V. Pino and J. L. Anderson, Anal. Chim. Acta, 2011, 687, 141-149.

172. L. Pang and J. F. Liu, J. Chromatogr. A, 2012, 1230, 8-14.

173. N. Reyes-Garces, E. Gionfriddo, G. A. Gomez-Rios, M. N. Alam, E. Boyaci, B. Bojko, V. Singh, J. Grandy and J. Pawliszyn, Anal. Chem., 2018, 90, 302-360.

174. F. David, N. Ochiai and P. Sandra, TRAC Trends Anal. Chem., 2019, 112, 102-111.

175. C. H. Yu and B. Hu, J. Sep. Sci., 2010, 33, 2176-2183.

176. W. A. W. Ibrahim, A. S. A. Keyon, N. Prastomo and A. Matsuda, J. Sol-Gel SCl. Technol., 2011, 59, 128-134.

177. N. Gilart, R. M. Marce, F. Borrull and N. Fontanals, Trends Anal. Chem., 2014, 54, 11-23. 
178. M. He, B. Chen and B. Hu, Anal. Bioanal. Chem., 2014, 406, 2001-2026.

179. E. van Hoeck, F. Canale, C. Cordero, S. Compernolle, C. Bicchi and P. Sandra, Anal. Bioanal. Chem., 2009, 393, 907-919.

180. M. C. Sampedro, M. A. Goicolea, N. Unceta, A. Sanchez-Ortega, Barrio and R.J., J. Sep. Sci., 2009, 32, 3449-3456.

181. N. Maslamani, E. Manandhar, D. K. Geremia and B. A. Logue, Anal. Chim. Acta, 2016, 941, 41-48.

182. A. H. Alluhayb and B. A. Logue, J. Chromatogr. A, 2017, 1518, 8-14.

183. N. Ochiai, K. Sasamoto, F. David and P. Sandra, J. Chromatogr. A, 2016, 1455, 45-56.

184. C. Yu, Q. Liu, L. Lan and B. Hu, J. Chromatogr. A, 2008, 1188, 124-131.

185. M. Kawaguchi, R. Ito, H. Nakazawa and A. Takatsu, Trends Anal. Chem., 2013, 45, 280-293.

186. N. Ochiai, K. Sasamoto, F. David and P. Sandra, J. Agric. Food Chem., 2018, 66, 7249-7255.

187. N. Gilart, R. M. Marce, P. A. G. Cormack, N. Fontanals and F. Borrull, J. Sep. Sci., 2014, 37, 2225-2232.

188. W. Fan, X. Mao, M. He, B. Chen and B. Hu, Anal. Bioanal. Chem., 2014, 406, 7261-7273.

189. J. P. Lambert, W. M. Mullett, E. Kwong and D. Lubda, J. Chromatogr. A, 2005, 1075, 43-49.

190. Y. Hu, J. Pan, K. Zhang, H. Lian and G. Li, Trends Anal. Chem., 2013, 43, 37-52.

191. Y. B. Luo, J. S. Cheng, Q. Ma, Y. Q. Feng and J. H. Li, Anal. Methods, 2011, 3, 92-98.

192. C. Hu, B. B. Chen, M. He and B. Hu, J. Chromatogr. A, 2013, 1300, 165-172.

193. J. L. Benede, A. Chisvert, D. L. Giokas and A. Salvador, J. Chromatogr. A, 2014, 1362, 25-33. 
Table 1. Selected SME application studies involving the use of extractants alternative to conventional VOSs.

\begin{tabular}{|c|c|c|c|c|c|c|c|}
\hline Sample & Analyte & Technique & Extractant $(\mu \mathrm{L})$ & $\begin{array}{l}\text { Extraction } \\
\text { time (min) }\end{array}$ & $\begin{array}{l}\text { Recovery } \\
\text { (RSD) } \\
(\%)\end{array}$ & $\begin{array}{l}\text { Instrumental } \\
\text { technique }\end{array}$ & Ref. \\
\hline Vegetable oils & Phenolic acid & UA-LLE $^{1}$ & $\begin{array}{l}\mathrm{ChCl}^{2}: \\
\text { ethylene glycol } \\
(1: 2)\end{array}$ & 15 & $95-113$ & LC-UV-Vis & 13 \\
\hline Sesame oil & Lignans & UA-LLE & $\begin{array}{l}\text { ChCl:p-cresol } \\
(1: 2)\end{array}$ & 35 & $97-120$ & LC-UV-Vis & 12 \\
\hline $\begin{array}{l}\text { Soft beverages } \\
\text { and tea }\end{array}$ & Caffeine & $\begin{array}{l}\text { Automated } \\
\text { LLE }\end{array}$ & $\begin{array}{l}\text { ChCl:phenol } \\
(1: 3)\end{array}$ & - & 101-104 & LC-UV-Vis & 26 \\
\hline $\begin{array}{l}\text { Leaves of } \\
\text { Chamaecypari } \\
\text { sobtuse }\end{array}$ & $\begin{array}{l}\text { Volatile } \\
\text { active } \\
\text { terpenoids }\end{array}$ & HS-SDME & $\begin{array}{l}\text { ChCl: ethylene } \\
\text { glycol (1:4) }\end{array}$ & 30 & $79-103$ & GC-FID ${ }^{3}$ & 27 \\
\hline Digested fish & $\begin{array}{l}\text { Trimethylami } \\
\text { ne }\end{array}$ & HS-SDME & $\begin{array}{l}\text { (CdSe/ZnS QDs)- } \\
{\left[\mathrm{C}_{6} \mathrm{MIM}\right]\left[\mathrm{PF}_{6}\right]^{4}} \\
(20)\end{array}$ & 2 & $92-106(4)$ & $\begin{array}{l}\text { Spectrofluorime } \\
\text { try }\end{array}$ & 28 \\
\hline $\begin{array}{ll}\text { Ground, lake } \\
\text { and } \\
\text { water }\end{array}$ & $\begin{array}{l}\text { Sulfonamide } \\
\mathrm{s}\end{array}$ & DI-SDME & $\begin{array}{l}{\left[\mathrm{C}_{8} \mathrm{MIM}\right]\left[\mathrm{PF}_{6}\right]^{5}} \\
(10)\end{array}$ & 20 & $64-116(4-10)$ & LC-UV & 29 \\
\hline $\begin{array}{l}\text { Digested dried } \\
\text { sausage }\end{array}$ & $\begin{array}{l}\text { 2-Amino-3,8- } \\
\text { dimethylimid } \\
\text { azo[4,5- } \\
\text { f]quinoxaline }\end{array}$ & DI-SDME & $\begin{array}{l}{[\mathrm{BMIM}]\left[\mathrm{PF}_{6}\right]^{6}+} \\
\mathrm{o}+\mathrm{NC}+\mathrm{C}-\mathrm{CNT} \\
\text { (4) }\end{array}$ & 30 & $90-95(3)$ & CE-DAD $^{7}$ & 30 \\
\hline $\begin{array}{l}\text { Ground, } \\
\text { surface and } \\
\text { wastewater }\end{array}$ & $\begin{array}{l}\text { Chloropheno } \\
\text { Is }\end{array}$ & DI-SDME & $\begin{array}{l}\text { Decanoic acid } \\
(30)\end{array}$ & 60 & $79-106(4-6)$ & LC-DAD & 31 \\
\hline River water & BTEX & $\mathrm{HF}(2) \mathrm{ME}$ & $\begin{array}{l}{[\mathrm{BMIM}]\left[\mathrm{PF}_{6}\right]} \\
\text { (8) }\end{array}$ & 30 & $90-112(1-5)$ & GC-FID & 29 \\
\hline
\end{tabular}




\begin{tabular}{|c|c|c|c|c|c|c|c|}
\hline $\begin{array}{l}\text { Rain and river } \\
\text { water }\end{array}$ & PAHs & $\mathrm{HF}(3) \mathrm{ME}$ & $\begin{array}{l}{[\mathrm{BMIM}] \mathrm{Cl}} \\
(10)\end{array}$ & 15 & $91-110(3-7)$ & LC-DAD & 32 \\
\hline $\begin{array}{l}\text { River and farm } \\
\text { waters }\end{array}$ & $\begin{array}{l}\text { Sulfonamide } \\
\mathrm{s}\end{array}$ & $\mathrm{HF}(3) \mathrm{ME}$ & $\begin{array}{l}{\left[\mathrm{C}_{8} \mathrm{MIM}\right]\left[\mathrm{PF}_{6}\right]} \\
\text { with } 14 \%(\mathrm{w} / \mathrm{v}) \\
\text { TOPO }\end{array}$ & 480 & $82-103(0.2-7)$ & LC-UV & 33 \\
\hline $\begin{array}{l}\text { River and tap } \\
\text { water }\end{array}$ & $\mathrm{OPPs}^{8}$ & SBME $^{9}$ & {$\left[\mathrm{C}_{8} \mathrm{MIM}\right]\left[\mathrm{PF}_{6}\right]$} & 60 & $\begin{array}{l}87-104 \\
(1-3) \\
\end{array}$ & LC-UV & 34 \\
\hline $\begin{array}{l}\text { Tap, lake and } \\
\text { fountain water }\end{array}$ & $\begin{array}{l}\text { Heterocyclic } \\
\text { insecticides }\end{array}$ & DLLME & $\begin{array}{l}{\left[\mathrm{C}_{6} \mathrm{MIM}\right]\left[\mathrm{PF}_{6}\right] \text { in }} \\
\mathrm{MeOH} \\
(52 \mathrm{mg})\end{array}$ & - & $79-106(4-11)$ & LC-DAD & 35 \\
\hline $\begin{array}{l}\text { River water } \\
\text { and urine }\end{array}$ & $\begin{array}{ll}\text { PCBs } & 10, \\
\text { PBDEs } & \end{array}$ & TC-DLLME $^{12}$ & $\begin{array}{l}{\left[\mathrm{C}_{8} \mathrm{MIM}\right]\left[\mathrm{PF}_{6}\right] \text { in }} \\
\mathrm{MeOH} \\
(40)\end{array}$ & 3 & $81-127(1-6)$ & LC-DAD & 36 \\
\hline $\begin{array}{l}\text { Infant formula } \\
\text { milk powder }\end{array}$ & $\begin{array}{l}\text { Sulfonamide } \\
\mathrm{s}\end{array}$ & $\bigcup_{13}^{\text {UA-TC-DLLME }}$ & $\begin{array}{l}{\left[\mathrm{C}_{6} \mathrm{MIM}\right]\left[\mathrm{PF}_{6}\right] \text { in }} \\
{\left[\mathrm{C}_{4} \mathrm{MIM}\right]\left[\mathrm{BF}_{4}\right]^{14}} \\
(70)\end{array}$ & 15 & $90-115(2-8)$ & LC-DAD & 37 \\
\hline $\begin{array}{l}\text { Tap, river and } \\
\text { waste water }\end{array}$ & $\begin{array}{l}\text { Aromatic } \\
\text { amines }\end{array}$ & DLLME & $\begin{array}{l}{[\mathrm{BMIM}]\left[\mathrm{PF}_{6}\right]} \\
(50)\end{array}$ & - & $93-106(6-10)$ & LC-UV & 38 \\
\hline $\begin{array}{l}\text { Red wine, fruit } \\
\text { juices }\end{array}$ & Sudan dyes & DLLME & $\begin{array}{l}{\left[\mathrm{C}_{6} \mathrm{MIM}\right]\left[\mathrm{PF}_{6}\right]} \\
(50)\end{array}$ & 10 & $68-108(1-6)$ & LC-DAD & 39 \\
\hline $\begin{array}{l}\text { Fruit juice, } \\
\text { vegetables }\end{array}$ & Pesticides & TC-DLLME & $\begin{array}{l}\text { ChCl:p- } \\
\text { chlorophenol } \\
(1: 8.5 ; 142)\end{array}$ & 5 & $\begin{array}{l}56-93 \\
(3-5)\end{array}$ & GC-FID & 40 \\
\hline $\begin{array}{l}\text { Food } \\
\text { supplements, } \\
\text { herbal tea } \\
\end{array}$ & Curcumin & VA-DLLME ${ }^{15}$ & $\begin{array}{l}\text { ChCl:phenol } \\
(1: 4 ; 400)\end{array}$ & 2 & $\begin{array}{l}96-102 \\
(1-6)\end{array}$ & LC-UV-Vis & 41 \\
\hline $\begin{array}{l}\text { Tap, lake and } \\
\text { waste water } \\
\text { and fruit juice }\end{array}$ & Fungicides & UA-DLLME & $\begin{array}{l}\text { Tween } 80 \\
(0.05 \mathrm{mg})\end{array}$ & 3 & $86-115(4-8)$ & $\begin{array}{l}\text { LC-DAD/ESI-MS } \\
16\end{array}$ & 42 \\
\hline $\begin{array}{l}\text { Water } \\
\text { samples }\end{array}$ & OPPs & VA-DLLME & Triton X-100 & 3 & $82-99(3-8)$ & GC-FPD $^{17}$ & 43 \\
\hline
\end{tabular}




\begin{tabular}{|l|l|l|l|l|l|l|l|}
\hline Urine & $\begin{array}{l}\text { Non- } \\
\text { steroidal } \\
\text { anti- } \\
\text { inflammator } \\
\text { y drugs }\end{array}$ & $\begin{array}{l}\text { In-syringe } \\
\text { DLLME }\end{array}$ & $\begin{array}{l}{\left[\mathrm{BMIM}^{\mathrm{MeOH}}\left[\mathrm{PF}_{6}\right] \text { in }\right.} \\
(250)\end{array}$ & 5 & $100-106$ (3-9) & LC-UV & 44 \\
& & & & & & \\
& & & & & \\
\hline
\end{tabular}

${ }^{1}$ UA-LLE: ultrasonic assisted-LLE; ${ }^{2} \mathrm{ChCl}$ : choline chloride; ${ }^{3} \mathrm{FID}$ : flame ionization detector; ${ }^{4}\left[\mathrm{C}_{6} \mathrm{MIM}\right]\left[\mathrm{PF}_{6}\right]$ : 1 -hexyl-3-methylimidazolium hexafluorophosphate; ${ }^{5}\left[\mathrm{C}_{8} \mathrm{MIM}\right]\left[\mathrm{PF}_{6}\right]$ : 1-octyl-3-methylimidazolium hexafluorophosphate; ${ }^{6}$ [BMIM][PF 6 : 1-benzyl-3-methyl-imidazolium hexafluorophosphate; ${ }^{7}$ DAD: diodo array detector; ${ }^{8}$ OPPs: Organophosphorus pesticides; ${ }^{9}$ SBME: solvent bar micro-extraction; ${ }^{10}$ PCBs: polychlorinated biphenyls; ${ }^{11}$ PBDEs: polybrominated diphenyl ethers; ${ }^{12}$ TC-DLLME: temperature controlled-DLLME; ${ }^{13}$ UA-TC-DLLME: ultrasonicassisted temperature controlled-DLLME; ${ }^{14}\left[\mathrm{C}_{4} \mathrm{MIM}_{\mathrm{MI}}\left[\mathrm{BF}_{4}\right]\right.$ : 1-butyl-3-methyl-imidazolium hexafluorophosphate; ${ }^{15}$ VA-DLLME: vortex assistedDLLME; ${ }^{16}$ ESI-MS: Electrospray ionization-mass spectrometry; ${ }^{17} \mathrm{FPD}$ : flame photometric detector. 


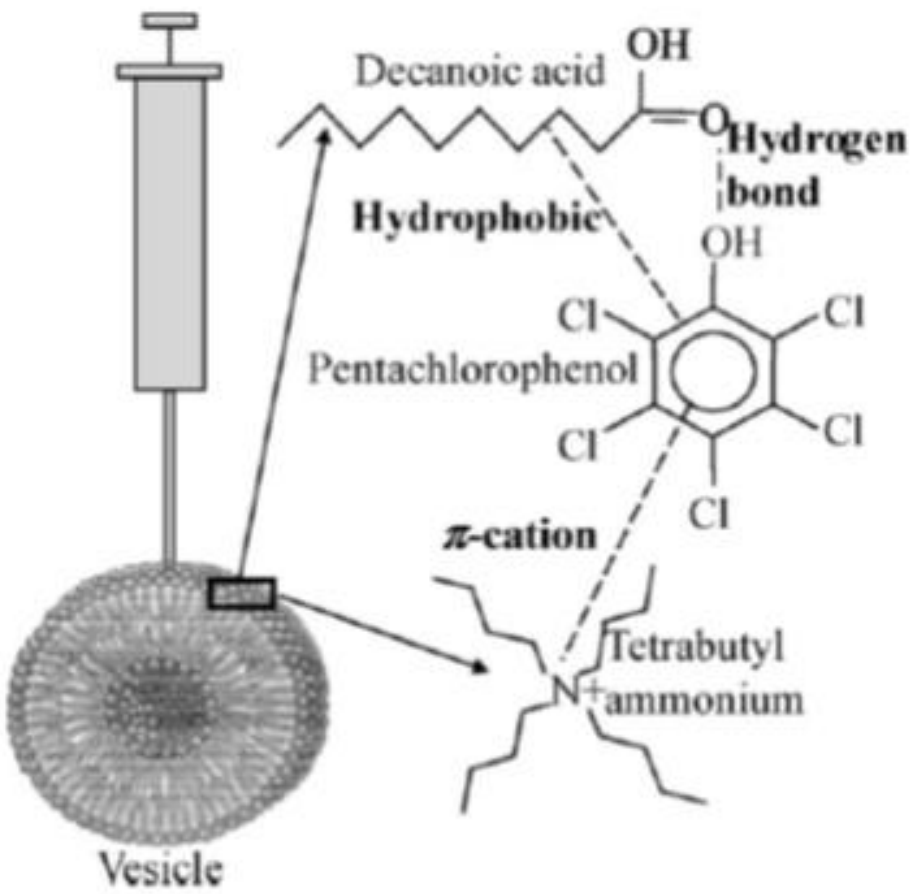

Figure 1. Schematic of the different intermolecular forces involved in the extraction of chlorophenols by SDME using decanoic acid vesicle-based coacervates. ${ }^{31}$ 


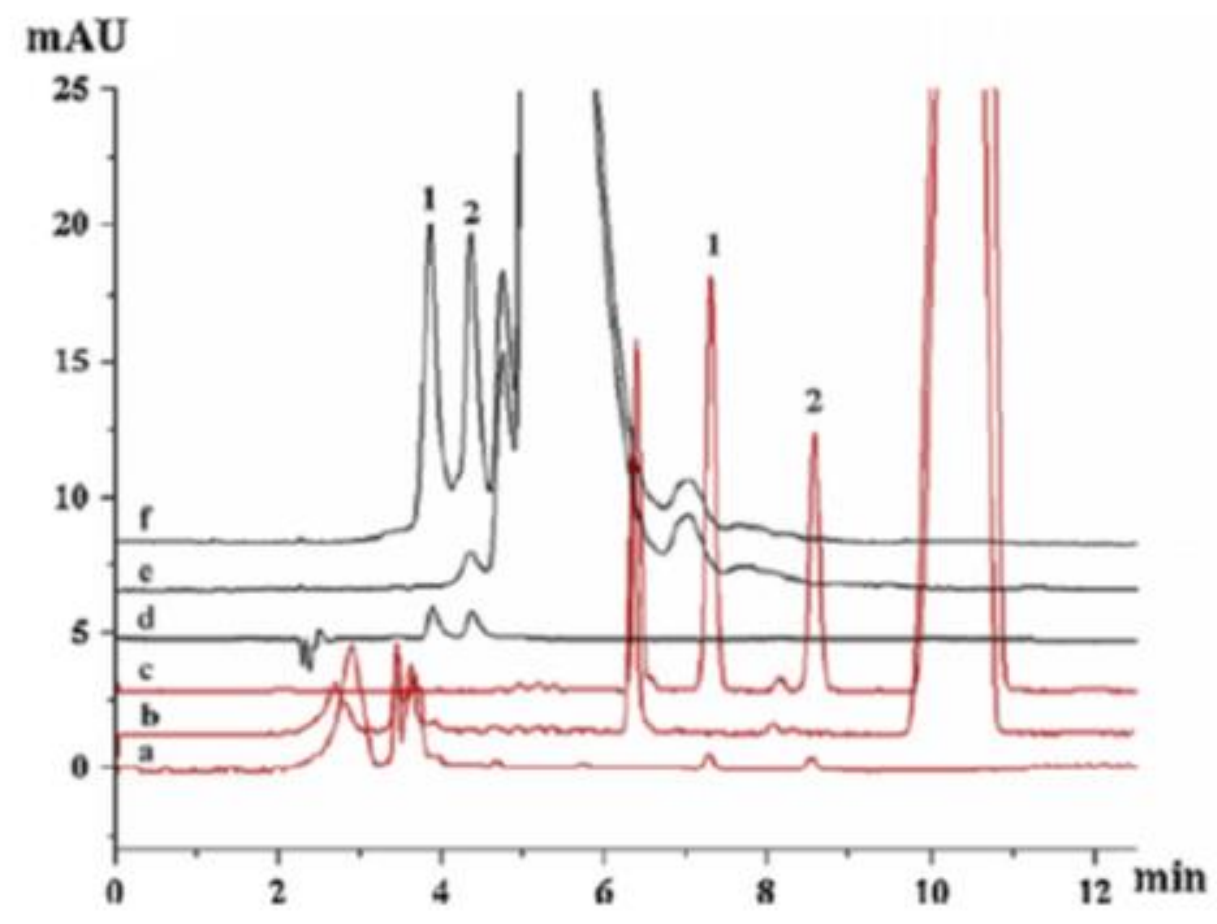

Figure 2. Chromatograms obtained for a spiked apple juice (a) before and (c) after UA-DLLME, and for a spiked East Lake water sample (d) before and after UA-DLLME; (b) blank apple sample and (e) blank East Lake water. Peak identification: (1) diethofencarb, and (2) pyrimethanil. ${ }^{42}$ 

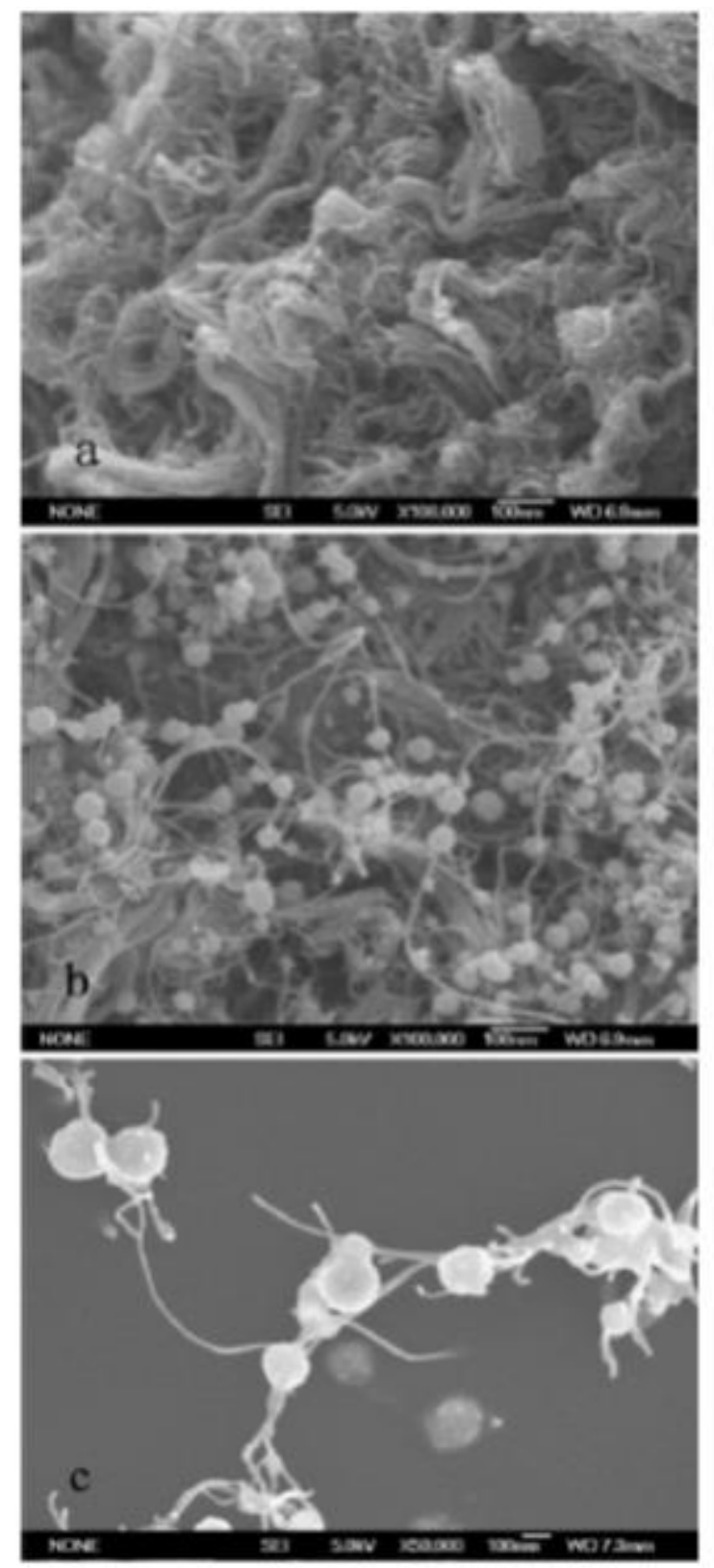

Figure 3. SEM images of (a) MWCNTs, (b) MWCNT@ $\mathrm{Fe}_{3} \mathrm{O}_{4}$, and (c) MWCNT@ $\mathrm{Fe}_{3} \mathrm{O}_{4}-\mathrm{MIPs}^{126}$ 

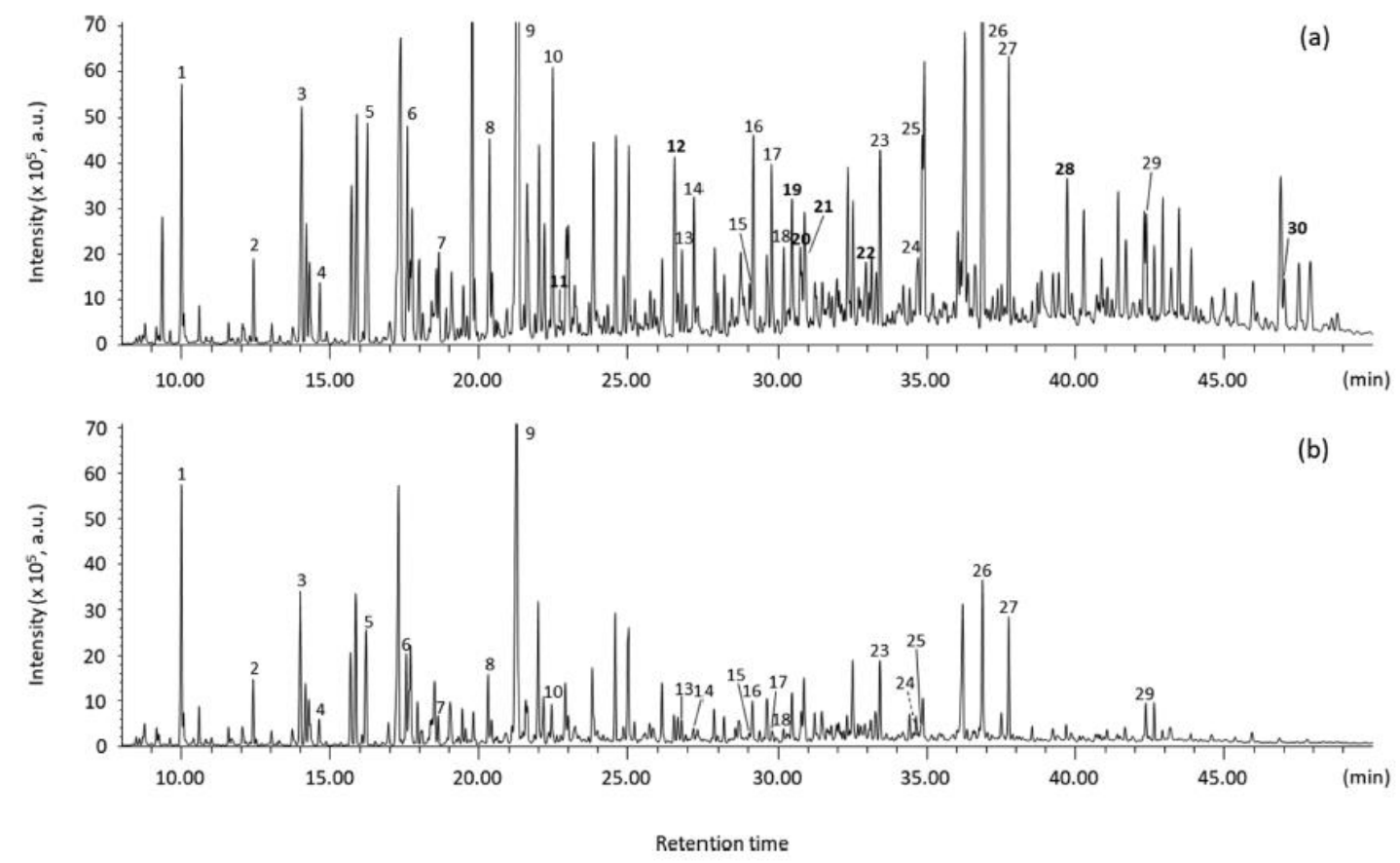

Figure 4. Comparison of TICs obtained for roasted green tea (Houji-cha) by (a) SA-SBSE and (b) conventional SBSE followed by TD-GC-MS. Peak identification: (1)1-ethyl pyrrole, (2) 2methylpyrazine, (3) 2,5-dimethylpyrazine, (4) 2,3-dimethylpyrazine, (5) 2,3,5-trimethylpyrazine, (6) furfural, (7) 2-acetylfuran, (8) 5-methyl furfural, (9) 1-ethyl-1H-pyrrole-2-carboxyaldehyde, (10) furfuryl alcohol, (11)isovaleric acid,(12)hexanoic acid, (13)guaiacol, (14) benzyl alcohol, (15) maltol, (16) 2-acetyl pyrrole, (17) phenol, (18) 2-formyl pyrrole, (19)furaneol,(20)methyl pyrrole-2carboxylate,(21)octanoic acid,(22)nonanoic acid, (23) 4-vinyl guaiacol, (24) 2,6-dimethoxyphenol, (25) 3-ethyl-4-methyl-1H-pyrrole-2,5-dione, (26) 4-vinyl phenol, (27) indole, (28)vanillin, (29) methoxy eugenol, and (30)raspberry ketone. Analytes 30-36 were not detected with conventional SBSE. ${ }^{186}$ 